\title{
Dyadic structure theorems for multiparameter function spaces
}

\author{
Ji Li, Jill Pipher and Lesley A. Ward
}

\begin{abstract}
We prove that the multiparameter (product) space BMO of functions of bounded mean oscillation can be written as the intersection of finitely many dyadic product BMO spaces, with equivalent norms, generalizing the one-parameter result of T. Mei. We establish the analogous dyadic structure theorems for the space VMO of functions of vanishing mean oscillation, for $A_{p}$ weights, for reverse-Hölder weights and for doubling weights. We survey several definitions of VMO and prove their equivalences, in the continuous, dyadic, one-parameter and product cases. In particular, we introduce the space of dyadic product VMO functions. We show that the weighted product Hardy space $H_{\omega}^{1}$ is the sum of finitely many translates of dyadic weighted $H_{\omega}^{1}$, for each $A_{\infty}$ weight $\omega$, and that the weighted strong maximal function is pointwise comparable to the sum of finitely many dyadic weighted strong maximal functions, for each doubling weight $\omega$. Our results hold in both the compact and non-compact cases.
\end{abstract}

\section{Introduction}

Function spaces and function classes are of considerable interest in harmonic analysis, since (i) a prototypical problem is to establish the boundedness of a singular integral operator from one function space to another, (ii) these operators also act on $L^{p}$ spaces weighted by functions in the $A_{p}$ or $R H_{p}$ function classes, and (iii) the density of the measure on the underlying space is often assumed to belong to the class of doubling weights. For brevity we use the term function spaces to refer to BMO, VMO, $H^{1}, A_{p}$ and $R H_{p}$ as well as the class of doubling weights. Dyadic function spaces offer a parallel setting in which calculation is often simpler, since one can exploit the geometry of the dyadic intervals. For instance, in [16] the

Mathematics Subject Classification (2010): Primary 42B35; Secondary 42B30, $42 \mathrm{~B} 25$.

Keywords: BMO, VMO, function spaces, doubling weights, $A_{p}$ weights, reverse-Hölder weights, Hardy space, maximal function, Carleson measures, multiparameter harmonic analysis, dyadic function spaces. 
John-Nirenberg inequality is proved by establishing a related inequality on certain dyadic cubes arising from a Calderón-Zygmund decomposition. For some recent developments in the theory of these function spaces, in particular in the multiparameter (product) setting, see for example [17] and [27].

In this paper we are concerned with a bridge between continuous and dyadic function spaces. The main theme is that for these function spaces, which are defined in terms of conditions that must hold uniformly on all intervals, the continuous version of the function space is an intersection of finitely many dyadic versions of the space, with equivalent norms, or in the case of $H^{1}$, a sum, with equivalent norms. There is also a related result for the maximal operator.

Our main results are the following dyadic structure theorems. We state the biparameter versions, for simplicity. The detailed statements and definitions are in the body of the paper.

Theorem A. The product BMO space is the intersection of finitely many dyadic product $\mathrm{BMO}$ spaces:

$\operatorname{BMO}(\mathbb{R} \otimes \mathbb{R})$

$$
=\mathrm{BMO}_{d, d}(\mathbb{R} \otimes \mathbb{R}) \cap \mathrm{BMO}_{d, \delta}(\mathbb{R} \otimes \mathbb{R}) \cap \mathrm{BMO}_{\delta, d}(\mathbb{R} \otimes \mathbb{R}) \cap \mathrm{BMO}_{\delta, \delta}(\mathbb{R} \otimes \mathbb{R}),
$$

with equivalent norms, for each real number $\delta$ that is far from dyadic rationals, in the sense of Definition 1.2 below. Here, for instance, $\mathrm{BMO}_{d, \delta}(\mathbb{R} \otimes \mathbb{R})$ is essentially a translate by $\delta$ in the second variable of the standard dyadic product space $\mathrm{BMO}_{d, d}(\mathbb{R} \otimes \mathbb{R})$. The corresponding intersection results hold for the one-parameter and product versions of $\mathrm{VMO}$, of $A_{p}$ with $1 \leq p \leq \infty$, of $R H_{p}$ with $1 \leq p \leq \infty$, and of doubling weights.

See Theorems 3.5, 3.6, 4.11, 7.1, and 7.3.

Theorem B. If $\omega$ is a product $A_{\infty}$ weight, then the weighted product Hardy space $H_{\omega}^{1}$ is the sum of finitely many weighted dyadic product Hardy spaces:

$$
H_{\omega}^{1}(\mathbb{R} \otimes \mathbb{R})=H_{d, d, \omega}^{1}(\mathbb{R} \otimes \mathbb{R})+H_{d, \delta, \omega}^{1}(\mathbb{R} \otimes \mathbb{R})+H_{\delta, d, \omega}^{1}(\mathbb{R} \otimes \mathbb{R})+H_{\delta, \delta, \omega}^{1}(\mathbb{R} \otimes \mathbb{R}),
$$

with equivalent norms, for each $\delta \in \mathbb{R}$ that is far from dyadic rationals.

If $\omega$ is a product doubling weight, then the weighted strong maximal function $M_{s, \omega}$ is pointwise comparable to the sum of finitely many weighted dyadic strong maximal functions:

$$
M_{s, \omega}(f) \sim M_{s}^{d, d, \omega}(f)+M_{s}^{d, \delta, \omega}(f)+M_{s}^{\delta, d, \omega}(f)+M_{s}^{\delta, \delta, \omega}(f),
$$

with implicit constants independent of $f \in L_{\text {loc }}^{1}(\mathbb{R} \otimes \mathbb{R})$, for each $\delta \in \mathbb{R}$ that is far from dyadic rationals.

See Theorems 5.2, 6.1, 8.1, and 8.2.

Remark 1.1. Theorems A and B extend to the $k$-parameter setting for arbitrary $k \in \mathbb{N}$, and in addition to the situation with higher-dimensional factors $\mathbb{R}^{n_{j}}$ replacing $\mathbb{R}$, for $1 \leq j \leq k$. Both generalizations require increasing the number of dyadic spaces in the intersection or sum. See Remarks 1.3, 1.4, and 1.5. 
The one-parameter dyadic function spaces $\mathrm{BMO}_{d}, \mathrm{VMO}_{d}$, etc. are defined using the usual grid $\mathcal{D}$ of dyadic intervals in $\mathbb{R}$. For $\delta \in \mathbb{R}$, let $\mathcal{D}^{\delta}$ denote the translate of $\mathcal{D}$ by $\delta$, modified as follows. Small dyadic intervals are simply translated by $\delta$. Large dyadic intervals are translated by $\delta$ and also by an additional amount which depends on the scale of the interval. See Section 2 for the precise construction of $\mathcal{D}^{\delta}$ and for a motivating example. It is then natural to define the $\delta$-dyadic function space $\mathrm{BMO}_{\delta}$ by requiring the BMO property to hold on intervals $I$ from the collection $\mathcal{D}^{\delta}$, and to define $\mathrm{VMO}_{\delta}, H_{\delta}^{1}, A_{p}^{\delta}, R H_{p}^{\delta}$ and $\delta$-dyadic doubling weights analogously. The biparameter dyadic function spaces are defined using $\mathcal{D} \times \mathcal{D}$, $\mathcal{D} \times \mathcal{D}^{\delta}, \mathcal{D}^{\delta} \times \mathcal{D}$, and $\mathcal{D}^{\delta} \times \mathcal{D}^{\delta}$.

In our structure theorems we take $\delta$ to be far from the dyadic rational numbers, in the following sense.

Definition 1.2. A real number $\delta$ is far from dyadic rationals if the distance from $\delta$ to each given dyadic rational $k / 2^{n}$ is at least some fixed multiple of $1 / 2^{n}$; that is, if

$$
\left|\delta-\frac{k}{2^{n}}\right| \geq \frac{C}{2^{n}} \quad \text { for all integers } n \text { and } k,
$$

where $C$ is a positive constant that may depend on $\delta$ but is independent of $n$ and $k$. Equivalently, the relative distance $d(\delta)$ from $\delta$ to the set of dyadic rational numbers is positive:

$$
d(\delta):=\inf \left\{2^{n}\left|\delta-\frac{k}{2^{n}}\right|: n \in \mathbb{Z}, k \in \mathbb{Z}\right\}>0 .
$$

For example, $\delta=1 / 3$ is far from dyadic rationals since $d(1 / 3)=1 / 3>0$. The set of all such $\delta$ is dense in $\mathbb{R}$ but has measure zero [19]. Note that $d(\delta+1)=d(\delta)$ for all $\delta \in \mathbb{R}$.

Remark 1.3. To reduce the amount of notation required, in the rest of the paper we work on $\mathbb{R}$ and $\mathbb{R} \otimes \mathbb{R}$. However, our results and proofs go through for $\mathbb{T}$ and $\mathbb{T} \otimes \mathbb{T}$, and for $\mathbb{R}^{m}, \mathbb{R}^{m_{1}} \otimes \mathbb{R}^{m_{2}}, \mathbb{T}^{m}$, and $\mathbb{T}^{m_{1}} \otimes \mathbb{T}^{m_{2}}$, and also for arbitrarily many factors in the multiparameter setting $\left(\mathbb{R}^{m_{1}} \otimes \cdots \otimes \mathbb{R}^{m_{k}}\right.$ and $\left.\mathbb{T}^{m_{1}} \otimes \cdots \otimes \mathbb{T}^{m_{k}}\right)$.

Remark 1.4. In the multiparameter setting, it is not necessary to choose the same $\delta$ for each direction. Take positive numbers $\delta_{1}, \delta_{2}, \ldots, \delta_{k}$ that are all far from dyadic rationals: $\min _{1 \leq j \leq k}\left\{d\left(\delta_{j}\right)\right\}>0$. Our proofs immediately generalize to show that, for example, $\mathrm{BMO}(\mathbb{R} \otimes \cdots \otimes \mathbb{R})$ is the intersection of the $2^{k}$ versions of dyadic product BMO formed by in the $j$ th direction choosing either $\mathcal{D}$ or $\mathcal{D}^{\delta_{j}}$ as the dyadic grid: namely $\mathrm{BMO}_{d, d, \ldots, d}, \mathrm{BMO}_{\delta_{1}, d, \ldots, d}, \mathrm{BMO}_{d, \delta_{2}, \ldots, d}, \ldots$, $\mathrm{BMO}_{\delta_{1}, \delta_{2}, \ldots, \delta_{k}}$. The same generalizations also hold for multiparameter VMO, $H^{1}$, $A_{p}, R H_{p}$, and doubling weights, and for the strong maximal function. For simplicity of notation, however, throughout the paper we have used the same $\delta$ for each direction: $\delta=\delta_{1}=\cdots=\delta_{k}$.

Remark 1.5. In [19], Mei established the one-parameter BMO result on the circle $\mathbb{T}$, and extended it to (one-parameter) $\mathbb{T}^{m}$, showing that $\operatorname{BMO}\left(\mathbb{T}^{m}\right)$ is the intersection of $m+1$ translates of the dyadic version of $\mathrm{BMO}\left(\mathbb{T}^{m}\right)$, and similarly to (oneparameter) $\mathbb{R}^{m}$, for $m \geq 1$. He also established the unweighted one-parameter $H^{1}$ 
result, and noted that the unweighted maximal function result follows from Doob's maximal inequality together with Proposition 2.1 of [19]. He mentions that John Garnett knew earlier that BMO coincides with the intersection of three translates of dyadic BMO, building on p. 417 of [11].

Remark 1.6. Mei's key proposition (see Proposition 2.1 of [19]) is a generalization of the so-called one-third trick. Namely, for each interval $Q$ there is an interval $I$ that contains $Q$, whose length is comparable to that of $Q$, and that belongs to either $\mathcal{D}$ or $\mathcal{D}^{1 / 3}$. The earliest reference we have found for this idea is on p. 339 of [21], although it was known earlier.

Remark 1.7. We compare the compact case (the circle $\mathbb{T}$ ) with the non-compact case (the real line $\mathbb{R})$. We define the circle to be the unit interval with endpoints identified: $\mathbb{T}:=[0,1] /(0 \sim 1)$. First, for the continuous function space BMO and for continuous $A_{p}, R H_{p}$ and doubling weights, there is only a small difference between the compact and non-compact cases: namely, the defining property is assumed to hold only on the intervals contained in $\mathbb{T}$ as opposed to on all intervals in $\mathbb{R}$. Second, for their dyadic versions $\left(\mathrm{BMO}_{d}, A_{p}^{d}, R H_{p}^{d}\right.$ and dyadic doubling weights), the same is true, with the additional difference that when considering translations by $\delta$, in the compact case $\mathbb{T}$ it suffices simply to translate each dyadic interval by $\delta$, while in the non-compact case $\mathbb{R}$, we translate intervals of length larger than 1 not only by the amount $\delta$ but also by an additional amount that depends on the scale, as mentioned above. See Section 2.

The differences for VMO are more subtle. First, for continuous VMO, in the compact case the definition of the subspace VMO of BMO involves a condition requiring the mean oscillation of the function to approach zero as the length of the interval goes to zero. In the non-compact case, one must impose two additional conditions controlling the mean oscillation over large intervals and over intervals that are far from the origin. (With this definition one retains the duality $\mathrm{VMO}^{*}=H^{1}$.) Second, for the dyadic non-compact case the same three oscillation conditions apply, and also when translating by $\delta$ we need the additional translations of intervals at large scales, as described in the preceding paragraph. See Section 4.

The same observations on the compact and non-compact cases apply to the oneparameter but higher-dimensional cases $\left(\mathbb{T}^{m}\right.$ and $\left.\mathbb{R}^{m}\right)$, and to the multiparameter case. We give the technical details in the body of the paper.

Remark 1.8. The overall approach of our proofs in Sections 3, 4, and 5 is as follows. We first prove the BMO intersection result, then from that together with checking the VMO conditions we deduce the VMO intersection result, which in turn implies the $H^{1}$ summation result by duality. We note that one could also obtain the same results by first proving the $H^{1}$ result by means of the atomic decompositions of the continuous and dyadic $H^{1}$ spaces, then deducing the BMO result by duality, and then proving the VMO result by the proof we give here. See the discussion in Section 5.

Remark 1.9. A second type of bridge between continuous and dyadic function classes, via averaging, is developed in the papers [12], [29], [23], [27], [24], [6]. 
Namely, a suitable family of functions in the dyadic version of a function space can be converted to a single function that belongs to the continuous version of the same space, via a translation-average (for BMO and $\mathrm{VMO}$ ) or a geometric-arithmetic average (for $A_{p}, R H_{p}$, and doubling weights). We do not discuss these matters further in the current paper.

As usual, the notation $A \lesssim B$ means $A \leq C B$ with a constant $C$ independent of all variables in $A$ and $B$, while $A \sim B$ means $A$ is comparable to $B$ in the sense that $A \lesssim B \lesssim A$.

The paper is organized as follows. In Section 2 we give the required background on dyadic and $\delta$-dyadic intervals. The key observation is that for intervals $Q$ and $I$ as in Proposition 2.1 of [19], and for a weight $\omega$ that is both dyadic doubling and $\delta$-dyadic doubling with $\delta$ far from dyadic rationals, the averages of $\omega$ on $Q$ and on $I$ are comparable. In Section 3 we give a new proof of Mei's BMO result, via the Carleson-measure characterization of BMO, and extend it to yield the multiparameter BMO dyadic structure theorem. In Section 4, we establish the equivalence of several definitions of $\mathrm{VMO}$, and do the same for the dyadic and product variants of VMO (Theorems 4.4, 4.6, 4.8, and 4.10), then prove the VMO structure theorem. We believe that the dyadic product $\mathrm{VMO}$ space $\mathrm{VMO}_{d, d}(\mathbb{R} \otimes \mathbb{R})$ has not appeared in the literature before.

In Section 5, we prove the dyadic structure theorem for the product Hardy space $H^{1}$, and discuss some consequences of the duality relations between BMO, $H^{1}$ and VMO. In Section 6 we prove that the multiparameter ("strong") maximal function is pointwise comparable to a sum of strong dyadic maximal functions. In Section 7 we prove our results for $A_{p}$ weights, $R H_{p}$ weights and doubling weights, including the extreme cases $A_{1}, A_{\infty}, R H_{1}$ and $R H_{\infty}$. We also establish weighted versions of the $A_{p}$ structure theorem and of Proposition 2.1 of [19]. Finally in Section 8 we present weighted versions of our results from Sections 5 and 6 on Hardy spaces and maximal functions.

\section{Dyadic intervals and $\delta$-dyadic intervals}

Let $\mathcal{D}=\mathcal{D}(\mathbb{R})$ denote the grid of dyadic intervals on $\mathbb{R}$ :

$$
\mathcal{D}(\mathbb{R})=\bigcup_{n \in \mathbb{Z}} \mathcal{D}_{n}(\mathbb{R}), \quad \text { where } \quad \mathcal{D}_{n}(\mathbb{R})=\left\{\left[\frac{k}{2^{n}}, \frac{k+1}{2^{n}}\right) \mid k \in \mathbb{Z}\right\} \quad \text { for each } n \in \mathbb{Z} .
$$

Define $\mathcal{D}(\mathbb{T})$ and $\mathcal{D}_{n}(\mathbb{T})$ on the circle $\mathbb{T}=[0,1] /(0 \sim 1)$ similarly.

For real $\delta$, we denote by $\mathcal{D}^{\delta}=\mathcal{D}^{\delta}(\mathbb{T})$ the translate to the right by $\delta$ of the dyadic grid on the circle $\mathbb{T}$, considered modulo 1 . Thus,

$$
\mathcal{D}^{\delta}(\mathbb{T}):=\{I+\delta \mid I \in \mathcal{D}(\mathbb{T})\}=\left\{\left[\left(\frac{k}{2^{n}}+\delta\right) \bmod 1,\left(\frac{k+1}{2^{n}}+\delta\right) \bmod 1\right) \mid k \in \mathbb{Z}\right\} .
$$

Finally, on the real line $\mathbb{R}$, following Remark 7 in [19], we include additional translations in the definition of the large-scale $\delta$-dyadic intervals in the $\delta$-dyadic 
$\operatorname{grid} \mathcal{D}^{\delta}(\mathbb{R})$. Our translates are not the same as Mei's. Specifically,

$$
\mathcal{D}^{\delta}(\mathbb{R})=\bigcup_{n \in \mathbb{Z}} D_{n}^{\delta}(\mathbb{R}),
$$

where for $n \geq 0$,

$$
D_{n}^{\delta}(\mathbb{R})=\left\{I+\delta \mid I \in \mathcal{D}_{n}(\mathbb{R})\right\}
$$

while for $n<0$ and $n$ even,

$$
D_{n}^{\delta}(\mathbb{R})=\left\{\left[\frac{k}{2^{n}}+\delta+\sum_{j=(n / 2)+1}^{0} 2^{-2 j}, \frac{k+1}{2^{n}}+\delta+\sum_{j=(n / 2)+1}^{0} 2^{-2 j}\right) \mid k \in \mathbb{Z}\right\} .
$$

These choices together with the nestedness property (namely, any two intervals in the same dyadic grid are either nested or disjoint) completely determine the collections $\mathcal{D}_{n}^{\delta}(\mathbb{R})$ for $n<0, n$ odd. We have translated by $\sum_{j=(n / 2)+1}^{0} 2^{-2 j}$, rather than by Mei's $\sum_{j=n+2}^{0} 2^{-j}$.

For example, for $n=-2$, the interval $[0,4)$ of length $2^{2}$ belongs to $\mathcal{D}_{-2}(\mathbb{R})$ while its translate $[\delta+1, \delta+5)$ belongs to $\mathcal{D}_{-2}^{\delta}(\mathbb{R})$, since $\sum_{j=0}^{0} 2^{-2 j}=1$. Similarly, for $n=-4$, the interval $[0,16)$ of length $2^{4}$ belongs to $\mathcal{D}_{-4}(\mathbb{R})$ while its translate $[\delta+5, \delta+21)$ belongs to $\mathcal{D}_{-4}^{\delta}(\mathbb{R})$, since $\sum_{j=-1}^{0} 2^{-2 j}=5$.

The key proposition in Mei's paper states that if $\delta$ is far from dyadic rationals, then for each interval $Q$ there is an interval $I$ containing $Q$, whose length $|I|$ is no greater than a fixed multiple of the length of $Q$, and which belongs either to the grid $\mathcal{D}$ of dyadic intervals or to the grid $\mathcal{D}^{\delta}$ of translated dyadic intervals.

Proposition 2.1 (Proposition 2.1 of [19]). Suppose $\delta \in(0,1)$ is far from dyadic rationals, as in condition (1.2). Then there is a constant $C(\delta)$ such that for each interval $Q$ in $\mathbb{R}$, there is an interval $I$ in $\mathbb{R}$ such that

(i) $Q \subset I$,

(ii) $|I| \leq C(\delta)|Q|$, and

(iii) $I \in \mathcal{D}$ or $I \in \mathcal{D}^{\delta}$.

The constant $C(\delta)$ can be taken to be $C(\delta)=2 / d(\delta)$.

Mei states this result on the circle $\mathbb{T}$ identified with $(0,2 \pi]$, with condition $(1.2)$ replaced by $d(\delta):=\inf \left\{2^{n}\left|\delta-k 2^{-n}\right| \mid n \geq 0, k \in \mathbb{Z}\right\}>0$, with $0<\delta<1$ and with the filtrations $\mathcal{D}(\mathbb{T})$ and $\mathcal{D}^{\delta}(\mathbb{T})$. For completeness we give a proof, stated on $\mathbb{R}$ and following Mei's proof. We also prove a weighted version below, during the proof of Lemma 7.2.

Proof of Proposition 2.1. Fix an interval $Q$ in $\mathbb{R}$. There exists an integer $n$ such that $d(\delta) 2^{-n-1} \leq|Q|<d(\delta) 2^{-n}$. Now we set $A_{n}=\left\{k \cdot 2^{n} \mid k \in \mathbb{Z}\right\}$ for every fixed $n$ and

$$
\text { (1) } A_{n}^{\delta}=\left\{\delta+k \cdot 2^{n} \mid k \in \mathbb{Z}\right\} \text { for } n \geq 0 \text {, }
$$


(2) $A_{n}^{\delta}=\left\{\delta+\sum_{j=(n / 2)+1}^{0} 2^{-2 j}+k \cdot 2^{n} \mid k \in \mathbb{Z}\right\}$ for $n<0, n$ even, and

(3) $A_{n}^{\delta}=\left\{\delta+\sum_{j=(n-1) / 2+1}^{0} 2^{-2 j}+k \cdot 2^{n} \mid k \in \mathbb{Z}\right\}$ for $n<0, n$ odd.

For any two distinct points $a, b \in A_{n} \cup A_{n}^{\delta}$, we have $|a-b| \geq d(\delta) 2^{-n}>|Q|$. Thus, at most one element of $A_{n} \cup A_{n}^{\delta}$ belongs to $Q$. Hence, $Q \cap A_{n}=\emptyset$ or $Q \cap A_{n}^{\delta}=\emptyset$. Therefore, $Q$ must be contained in some dyadic interval $I \in \mathcal{D}$ or in some $\delta$-dyadic interval $I \in \mathcal{D}^{\delta}$, and $|I|=2^{-n} \leq(2 / d(\delta))|Q|$.

As an aside, we note that the corresponding result holds for intervals $I^{\prime}$ contained in $Q$.

A weight is a nonnegative locally integrable function $\omega$ on $\mathbb{R}$. As usual, by a doubling weight we mean a weight such that $\omega(\widetilde{Q}) \leq C \omega(Q)$ with a positive constant $C$ independent of $Q$, where the double $\widetilde{Q}$ of an interval $Q$ is the interval with the same midpoint as $Q$ and twice the length of $Q:|\widetilde{Q}|=2|Q|$. Here as usual $\omega(Q):=\int_{Q} \omega(x) d x$. A dyadic doubling weight satisfies the corresponding condition $\omega(\widetilde{I}) \leq C \omega(I)$, where $\widetilde{I}$ is the dyadic parent of a dyadic interval $I \in \mathcal{D}$ : $\widetilde{I} \supset I, \widetilde{I} \in \mathcal{D}$, and $|\widetilde{I}|=2|I|$, so $I$ is either the left or the right half of $\widetilde{I}$. The $\delta$-dyadic weights are defined similarly, using $\mathcal{D}^{\delta}$ in place of $\mathcal{D}$.

We will frequently use the following consequence of Proposition 2.1.

Lemma 2.2. Let $\delta$ be far from dyadic rationals. Let $\omega$ be a weight that is both dyadic $\mathcal{D}$-doubling and dyadic $\mathcal{D}^{\delta}$-doubling with constant $C_{\mathrm{dy}}$. Then given intervals $Q$ and $I$ as in Proposition 2.1, the averages of $\omega$ on $Q$ and on $I$ are comparable:

$$
\left(C_{\mathrm{dy}}\right)^{-\log _{2}(4 C(\delta))} f_{I} \omega \leq f_{Q} \omega \leq C(\delta) f_{I} \omega .
$$

Proof of Lemma 2.2. Let $N$ be the unique integer such that $2^{N-1}<C(\delta) \leq 2^{N}$. Then $N+1<\log _{2}(4 C(\delta))$, and

$$
\frac{|I|}{2^{N+1}} \leq \frac{|I|}{2 C(\delta)} \leq \frac{|Q|}{2}
$$

Therefore, considering the $2^{N+1}$ pairwise disjoint subintervals $J$ of $I$ of length $|J|=|I| / 2^{N+1}$ (these $J$ are dyadic if $I \in \mathcal{D}, \delta$-dyadic if $I \in \mathcal{D}^{\delta}$ ), we see that one of these intervals $J$ must be completely contained in $Q$. For this $J$, we have

$$
\begin{aligned}
f_{I} \omega=\frac{1}{|I|} \int_{I} \omega & \leq\left(C_{\mathrm{dy}}\right)^{N+1} \frac{1}{|I|} \int_{J} \omega \\
& \leq\left(C_{\mathrm{dy}}\right)^{\log _{2}(4 C(\delta))} \frac{1}{|Q|} \int_{Q} \omega=\left(C_{\mathrm{dy}}\right)^{\log _{2}(4 C(\delta))} f_{Q} \omega .
\end{aligned}
$$

Moreover, since $Q \subset I,|I| \leq C(\delta)|Q|$, and $\omega \geq 0$, we have

$$
f_{Q} \omega=\frac{1}{|Q|} \int_{Q} \omega \leq \frac{C(\delta)}{|I|} \int_{I} \omega=C(\delta) f_{I} \omega .
$$


Example 2.3. This example illustrates the difference between $\mathbb{T}$ and $\mathbb{R}$ with regard to translations, and the need in the definition above of the $\delta$-dyadic intervals in $\mathcal{D}^{\delta}(\mathbb{R})$ for the global translations at certain scales. First, view the circle $\mathbb{T}$ as $[0,1]$ with the endpoints identified. Take $\delta>0$ with $d(\delta)>0$. By definition of $d(\delta)$, we have $d(\delta) \leq \delta$. Let $Q$ be an interval in $\mathbb{T}$ containing 0 and $\delta$ as interior points. Then $|Q| \geq \delta$. It follows that the properties asserted in Proposition 2.1 hold for the choice $I=\mathbb{T}$, since $Q \subset \mathbb{T}, \mathbb{T} \in \mathcal{D}$, and $|\mathbb{T}|=1 \leq|Q| / \delta$ so that

$$
\frac{|\mathbb{T}|}{|Q|} \leq \frac{1}{\delta} \leq \frac{1}{d(\delta)} \leq \frac{2}{d(\delta)}=C(\delta) .
$$

On the real line $\mathbb{R}$, however, the situation is different. Take the usual collection $\mathcal{D}$ of dyadic intervals, take any positive $\delta$ and let $\mathcal{D}^{\delta, \#}$ denote the translation to the right by $\delta$ of the dyadic grid $\mathcal{D}$, so that $I^{\#} \in \mathcal{D}^{\delta \text {, }}$ if and only if $I^{\#}=I+\delta$ for some $I \in \mathcal{D}$. Let $Q$ be an interval containing both 0 and $\delta$ in its interior.

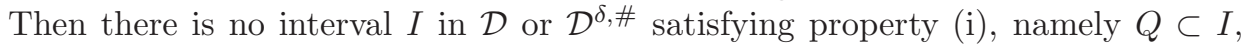
of Proposition 2.1, since dyadic intervals do not have 0 as an interior point and intervals in $\mathcal{D}^{\delta, \#}$ do not have $\delta$ as an interior point.

The use in $\mathcal{D}^{\delta}$ of the additional translations at scale $2^{-n}$ for all even $n<0$ ensures that the conclusion of Lemma 2.2 does hold for the intervals $Q \subset \mathbb{R}$ in this example.

\section{BMO and product BMO}

We extend Mei's BMO result to the biparameter case. We begin by recalling some observations and background results; for details see [5], [26]. Next we give a new proof of Mei's one-parameter result, still using Mei's proposition but expressing BMO in terms of Carleson measures. Then we extend this proof to the multiparameter case.

Proposition 3.1. Let $\psi \in C_{c}^{\infty}(\mathbb{R})$ be a smooth function, supported in the interval $[-1,1]$, such that $\int \psi(t) d t=0$. For $y>0$ let $\psi_{y}(t):=\frac{1}{y} \psi\left(\frac{t}{y}\right)$. For $t \in \mathbb{R}$ and $y>0$ let $I_{t, y}:=[t-y, t+y]$. Then,

(i) if $(t, y) \in T\left(I_{0}\right)$ then $I_{t, y} \subset 3 I_{0}$, where $3 I_{0}$ is the interval with the same midpoint as $I_{0}$ and length $\left|3 I_{0}\right|=3\left|I_{0}\right|$,

(ii) $\operatorname{supp} \psi_{y} \subset[-y, y]$,

(iii) $\operatorname{supp} \psi_{y}(t-\cdot) \subset I_{t, y}$,

(iv) for $I \in \mathcal{D}$ or $I \in \mathcal{D}^{\delta}$, and for the Haar function $h_{I}$, if $I \cap I_{t, y}=\emptyset$ then $h_{I} * \psi_{y}(t)=0$, and

(v) if $I_{t, y} \subset Q_{l}$ or $I_{t, y} \subset Q_{r}$, where $Q_{l}$ and $Q_{r}$ are the left and right halves respectively of an interval $Q$, then $h_{I} * \psi_{y}(t)=0$.

We omit the (elementary) proofs, except to note that part (v) holds since $h_{Q}$ is constant on each of $Q_{l}$ and $Q_{r}, \psi_{y}$ is supported in $I_{t, y}$, and $\int_{\mathbb{R}} \psi=0$. Now 
we impose an additional condition (the Calderón-Torchinsky condition) on $\psi$ as follows: there exists a constant $C_{\psi}$ such that for each $\xi \neq 0$,

$$
\int_{0}^{\infty} \frac{|\widehat{\psi}(\xi t)|^{2}}{t} d t \leq C_{\psi}
$$

If $f \in L^{2}(\mathbb{R})$, then $\|f\|_{L^{2}(\mathbb{R})}^{2}=\sum_{I \in \mathcal{D}}\left(f, h_{I}\right)^{2}$. Moreover, the following standard Littlewood-Paley $L^{2}$ estimate holds:

$$
\iint_{(t, y) \in \mathbb{R} \otimes \mathbb{R}_{+}}\left|f * \psi_{y}(t)\right|^{2} \frac{d t d y}{y} \leq C_{\psi}\|f\|_{L^{2}(\mathbb{R})}^{2},
$$

where $\psi \in C_{\mathrm{c}}^{\infty}(\mathbb{R}), \int \psi=0$, and $\psi$ satisfies (3.1).

Definition 3.2. A locally integrable function $f$ belongs to the dyadic BMO space $\mathrm{BMO}_{d}(\mathbb{R})$ if there is a constant $C$ such that

$$
\|f\|_{\mathrm{BMO}_{d}(\mathbb{R})}:=\sup _{I \in \mathcal{D}} f_{I}\left|f(x)-f_{I}\right| d x<\infty,
$$

where $f_{I}:=f_{I} f$.

It follows from the John-Nirenberg theorem that for each $p>1$, the expression

$$
\|f\|_{\mathrm{BMO}_{d, p}(\mathbb{R})}:=\sup _{I \in \mathcal{D}}\left(f_{I}\left|f(x)-f_{I}\right|^{p} d x\right)^{1 / p}
$$

is comparable to $\|f\|_{\mathrm{BMO}_{d}(\mathbb{R})}$; see Corollary 2.3 on p. 233 of [11].

Here is an equivalent definition of $\mathrm{BMO}_{d}(\mathbb{R})$ in terms of dyadic Carleson measures.

Definition 3.3. A locally integrable function $f$ belongs to the dyadic BMO space $\mathrm{BMO}_{d}(\mathbb{R})$ if there is a constant $C$ such that for all dyadic intervals $J$,

$$
\sum_{I \in \mathcal{D}, I \subset J}\left(f, h_{I}\right)^{2} \leq C|J| .
$$

Here $\left(f, h_{I}\right)$ is the Haar coefficient of $f$ with respect to the Haar function $h_{I}$ of $I \in \mathcal{D}$.

The smallest constant $C$ in condition (3.4) is comparable to $\|f\|_{\mathrm{BMO}_{d}(\mathbb{R})}^{2}$.

We note that if in Definition 3.3 we allow $J$ to range over all intervals in $\mathbb{R}$, not only dyadic intervals in $\mathbb{R}$, we recover the same dyadic BMO space $\mathrm{BMO}_{d}(\mathbb{R})$, with comparable norms. This observation follows from the fact that the sum in inequality (3.4) is over only dyadic intervals $I$, together with the observation that for each interval $Q \subset \mathbb{R}$, dyadic or not, there are two adjacent dyadic intervals $J_{1}$ and $J_{2}$ such that $Q \subset J_{1} \cup J_{2}$ and

$$
\frac{\left|J_{1}\right|}{2}=\frac{\left|J_{2}\right|}{2}<|Q| \leq\left|J_{1}\right|=\left|J_{2}\right| .
$$

For $\delta \in \mathbb{R}$ we define $\mathrm{BMO}_{\delta}(\mathbb{R})$ similarly, in terms of both averages and Carleson conditions, with respect to the collection $\mathcal{D}^{\delta}$ from Section 2. 
Definition 3.4. For $f \in L_{\text {loc }}^{1}(\mathbb{R})$ and $J$ a dyadic interval, define the projection $P_{J}$ of $f$ by

$$
P_{J} f(x):=\sum_{I \in \mathcal{D}, I \subset J}\left(f, h_{I}\right) h_{I}(x) .
$$

We now re-prove the dyadic structure theorem for one-parameter BMO.

Theorem 3.5. Suppose $\delta \in \mathbb{R}$ is far from dyadic rationals, in the sense of condition $(1.2)$. Then $\mathrm{BMO}(\mathbb{R})=\mathrm{BMO}_{d}(\mathbb{R}) \cap \mathrm{BMO}_{\delta}(\mathbb{R})$. Moreover, the norms are comparable:

$$
\begin{aligned}
\max \left\{\|f\|_{\mathrm{BMO}_{d}(\mathbb{R})},\|f\|_{\mathrm{BMO}_{\delta}(\mathbb{R})}\right\} & \\
& \leq\|f\|_{\mathrm{BMO}(\mathbb{R})} \leq(C \cdot C(\delta))^{1 / 2} \max \left\{\|f\|_{\mathrm{BMO}_{d}(\mathbb{R})},\|f\|_{\mathrm{BMO}_{\delta}(\mathbb{R})}\right\},
\end{aligned}
$$

where $C$ depends only on $C_{\psi}$ in condition (3.1).

Proof. The inclusion $\mathrm{BMO}(\mathbb{R}) \subset \mathrm{BMO}_{d}(\mathbb{R}) \cap \mathrm{BMO}_{\delta}(\mathbb{R})$ is an immediate consequence of the definition

$$
\operatorname{BMO}(\mathbb{R})
$$

$$
:=\left\{f \in L_{\mathrm{loc}}^{1}(\mathbb{R}):\|f\|_{*}=\|f\|_{\mathrm{BMO}(\mathbb{R})}:=\sup _{Q} \frac{1}{|Q|} \int_{Q}\left|f(x)-f_{Q}\right| d x<\infty\right\}
$$

via averages $f_{Q}:=(1 /|Q|) \int_{Q} f$, since for $\mathrm{BMO}_{d}(\mathbb{R})$ and $\mathrm{BMO}_{\delta}(\mathbb{R})$ the supremum is over fewer intervals than for $\mathrm{BMO}(\mathbb{R})$. Further, $\max \left\{\|f\|_{\mathrm{BMO}_{d}(\mathbb{R})},\|f\|_{\mathrm{BMO}_{\delta}(\mathbb{R})}\right\} \leq$ $\|f\|_{\mathrm{BMO}(\mathbb{R})}$.

Now we prove the other inclusion. Our proof, which relies on the Carlesonmeasure characterization of $\mathrm{BMO}(\mathbb{R})$, is more complicated than the original proof in [19]. We give this proof because it readily generalizes to the multiparameter case (Theorem 3.6). Suppose $f$ belongs to $\mathrm{BMO}_{d}(\mathbb{R}) \cap \mathrm{BMO}_{\delta}(\mathbb{R})$. Choose $\psi$ as in Proposition 3.1 and satisfying (3.1). We must show that there is a positive constant $C$ such that for every interval $I_{0}$,

$$
\iint_{T\left(I_{0}\right)}\left|f * \psi_{y}(t)\right|^{2} \frac{d t d y}{y} \leq C\left|I_{0}\right|,
$$

for the Carleson box $T\left(I_{0}\right):=\left\{(t, y): t \in I_{0}, 0<y<\left|I_{0}\right|\right\}$.

Fix an interval $I_{0} \subset \mathbb{R}$. For each point $(t, y)$ in $T\left(I_{0}\right)$, let $I_{t, y}:=(t-y, t+y)$ be the interval of length $2 y$ centered at $t$. By Proposition 2.1, we may choose an interval $I_{t, y}^{*}$ such that $I_{t, y} \subset I_{t, y}^{*},\left|I_{t, y}^{*}\right| \leq C(\delta)\left|I_{t, y}\right|$, and either $I_{t, y}^{*} \in \mathcal{D}$ or $I_{t, y}^{*} \in \mathcal{D}^{\delta}$. Let

$$
\mathcal{F}_{1}:=\left\{(t, y) \in T\left(I_{0}\right) \mid I_{t, y}^{*} \in \mathcal{D}\right\}, \quad \text { and } \quad \mathcal{F}_{2}:=\left\{(t, y) \in T\left(I_{0}\right) \mid I_{t, y}^{*} \in \mathcal{D}^{\delta}\right\} .
$$

So $T\left(I_{0}\right)=\mathcal{F}_{1} \cup \mathcal{F}_{2}$, and $\mathcal{F}_{1} \cap \mathcal{F}_{2}=\emptyset$. Now we have

$$
\begin{aligned}
& \iint_{(t, y) \in T\left(I_{0}\right)}\left|f * \psi_{y}(t)\right|^{2} \frac{d t d y}{y} \\
&=\iint_{(t, y) \in \mathcal{F}_{1}}\left|f * \psi_{y}(t)\right|^{2} \frac{d t d y}{y}+\iint_{(t, y) \in \mathcal{F}_{2}}\left|f * \psi_{y}(t)\right|^{2} \frac{d t d y}{y}=: G_{1}+G_{2} .
\end{aligned}
$$


It suffices to control the term $G_{1}$, since the estimate for the term $G_{2}$ is similar. Replacing $f$ by its Haar expansion, we see that

$$
f * \psi_{y}(t)=\sum_{I \in \mathcal{D}}\left(f, h_{I}\right) h_{I} * \psi_{y}(t)=\sum_{I \in \mathcal{D}, I \cap I_{t, y} \neq \emptyset}\left(f, h_{I}\right) h_{I} * \psi_{y}(t),
$$

since by Proposition 3.1 (iv), $h_{I} * \psi_{y}(t)$ can only be nonzero if $I \cap I_{t, y} \neq \emptyset$.

For each $(t, y) \in \mathcal{F}_{1}$, we have $I_{t, y} \subset 3 I_{0}$, by Proposition 3.1 (i).

Fix $(t, y) \in \mathcal{F}_{1}$. We split the sum in equation (3.7) at the scale of $2^{N}\left|3 I_{0}\right|$, where $N>0$ is a constant to be determined later but independent of $f, t, y$ and $I_{0}$. Let $k_{0}$ be the unique integer such that

$$
2^{-k_{0}} \leq\left|3 I_{0}\right|<2^{-k_{0}+1}
$$

Now,

$$
\begin{aligned}
f * \psi_{y}(t)= & \sum_{k=k_{0}-N-1}^{\infty} \sum_{I \in \mathcal{D}_{k}, I \cap I_{t, y} \neq \emptyset}\left(f, h_{I}\right) h_{I} * \psi_{y}(t) \\
& +\sum_{k=-\infty}^{k_{0}-N-2} \sum_{I \in \mathcal{D}_{k}, I \cap I_{t, y} \neq \emptyset}\left(f, h_{I}\right) h_{I} * \psi_{y}(t)=: g_{11}+g_{12} .
\end{aligned}
$$

For the sum $g_{12}$ : We first show that each term in the sum $g_{12}$ over large intervals is zero, if $N$ is chosen appropriately. Let $N$ be the unique integer such that

$$
2^{N} \leq 2 C(\delta)<2^{N+1} .
$$

(Note that $N \geq 2$, since $d(\delta)<1$ and so $2 C(\delta)=4 / d(\delta)>2^{2}$.) We will use the right-hand inequality in (3.8) for our estimate of $g_{12}$, and the left-hand inequality for $g_{11}$.

If the interval $I$ appears in the sum $g_{12}$, we have

$$
|I| \geq 2^{-k_{0}+N+2}>2^{N+1}\left|3 I_{0}\right|>2 C(\delta)\left|I_{t, y}\right| \geq 2\left|I_{t, y}^{*}\right| .
$$

Since the intervals $I$ and $I_{t, y}^{*}$ both belong to the same dyadic grid $\mathcal{D}$ and $|I|>\left|I_{t, y}^{*}\right|$, it follows that either $I$ and $I_{t, y}^{*}$ are disjoint or $I \supsetneqq I_{t, y}^{*}$. If the former, then $h_{I} * \psi_{y}(t)=0$. If the latter, then since $I_{t, y} \subset I_{t, y}^{*} \varsubsetneqq I$ we see that $I_{t, y}$ is contained in either the left half of $I$ or the right half of $I$, and so by Proposition $3.1(\mathrm{v})$, $h_{I} * \psi_{y}(t)=0$. Thus the sum $g_{12}$ is zero.

For the sum $g_{11}$ : For each interval $I$ that appears in the sum $g_{11}$, we have $|I| \leq 2^{-k_{0}+N+1} \leq 2^{N+1}\left|3 I_{0}\right|$ and $I \cap 3 I_{0} \neq \emptyset$. It follows that each such interval $I$ is contained in the interval

$$
J_{0}:=2^{N+1} 9 I_{0}
$$

that has the same midpoint as $I_{0}$ and length $\left|J_{0}\right|=2^{N+1}\left|9 I_{0}\right|$. 
Then

$$
\begin{aligned}
g_{11} & :=\sum_{k=k_{0}-N-1}^{\infty} \sum_{I \in \mathcal{D}_{k}, I \cap I_{t, y} \neq \emptyset}\left(f, h_{I}\right) h_{I} * \psi_{y}(t) \\
& =\sum_{k=k_{0}-N-1}^{\infty} \sum_{I \in \mathcal{D}_{k}, I \subset J_{0}}\left(f, h_{I}\right) h_{I} * \psi_{y}(t)=\sum_{I \in \mathcal{D}, I \subset J_{0}}\left(f, h_{I}\right) h_{I} * \psi_{y}(t) .
\end{aligned}
$$

The third equality holds because if $I \subset J_{0}, I \in \mathcal{D}_{k}$ and $k<k_{0}-N-1$, then $h_{I} * \psi_{y}(t)=0$ by Proposition 3.1 and the argument for $g_{12}$ above.

As a consequence, and applying the Littlewood-Paley $L^{2}$ estimate (3.2) and the Carleson condition (3.4) for $f \in \mathrm{BMO}_{d}(\mathbb{R})$ and the interval $J_{0}$, we see that

$$
\begin{aligned}
G_{1} & =\iint_{\mathcal{F}_{1}}\left|g_{11}\right|^{2} \frac{d t d y}{y} \leq \iint_{\mathcal{F}_{1}}\left|\sum_{I \in \mathcal{D}, I \subset J_{0}}\left(f, h_{I}\right) h_{I} * \psi_{y}(t)\right|^{2} \frac{d t d y}{y} \\
& =\iint_{\mathcal{F}_{1}}\left|P_{J_{0}} f * \psi_{y}(t)\right|^{2} \frac{d t d y}{y} \leq \iint_{(t, y) \in \mathbb{R} \otimes \mathbb{R}_{+}}\left|P_{J_{0}} f * \psi_{y}(t)\right|^{2} \frac{d t d y}{y} \\
& \leq C\left\|P_{J_{0}} f\right\|_{L^{2}(\mathbb{R})}^{2}=C \sum_{I \in \mathcal{D}}\left(P_{J_{0}} f, h_{I}\right)^{2}=C \sum_{I \in \mathcal{D}, I \subset J_{0}}\left(f, h_{I}\right)^{2} \\
& \leq C\left|J_{0}\right|\|f\|_{\mathrm{BMO}_{d}(\mathbb{R})}^{2} \leq C 2^{N+1}\left|I_{0}\right|\|f\|_{\mathrm{BMO}_{d}(\mathbb{R})}^{2} \leq C \cdot C(\delta)\left|I_{0}\right|\|f\|_{\mathrm{BMO}_{d}(\mathbb{R})}^{2},
\end{aligned}
$$

where $C$ depends only on the $C_{\psi}$ in (3.1).

In the same way, we obtain the estimate

$$
\left(G_{2}\right) \leq C \cdot C(\delta)\left|I_{0}\right|\|f\|_{\mathrm{BMO}_{\delta}(\mathbb{R})}^{2} .
$$

Therefore, as required, inequality (3.6) holds, and

$$
\|f\|_{\mathrm{BMO}(\mathbb{R})} \lesssim C(\delta)^{1 / 2} \max \left\{\|f\|_{\mathrm{BMO}_{d}(\mathbb{R})},\|f\|_{\mathrm{BMO}_{\delta}(\mathbb{R})}\right\} .
$$

We now turn to the product setting. For simplicity we discuss the biparameter case.

A locally integrable function $f$ on $\mathbb{R} \otimes \mathbb{R}$ belongs to the product BMO space $\mathrm{BMO}(\mathbb{R} \otimes \mathbb{R})$ if there exists a positive constant $C$ such that for every open set $\Omega \subset \mathbb{R} \otimes \mathbb{R}$ with finite measure, the following inequality holds:

$$
\iint_{T(\Omega)}\left|f * \psi_{y_{1}} \psi_{y_{2}}\left(t_{1}, t_{2}\right)\right|^{2} \frac{d t_{1} d y_{1} d t_{2} d y_{2}}{y_{1} y_{2}} \leq C|\Omega| .
$$

Here $T(\Omega):=\left\{\left(t_{1}, y_{1}, t_{2}, y_{2}\right) \mid I_{t_{1}, y_{1}} \times I_{t_{2}, y_{2}} \subset \Omega\right\}$ is the Carleson tent on $\Omega$, and for $\psi$ as in the one-parameter proof above, $\psi_{y_{1}} \psi_{y_{2}}\left(t_{1}, t_{2}\right):=y_{1}^{-1} y_{2}^{-1} \psi\left(t_{1} / y_{1}\right) \psi\left(t_{2} / y_{2}\right)$. The smallest such $C$ is comparable to $\|f\|_{\mathrm{BMO}(\mathbb{R} \otimes \mathbb{R})}^{2}$.

Next we mention the four types of dyadic product BMO spaces $\mathrm{BMO}_{d, d}(\mathbb{R} \otimes \mathbb{R})$, $\mathrm{BMO}_{d, \delta}(\mathbb{R} \otimes \mathbb{R}), \mathrm{BMO}_{\delta, d}(\mathbb{R} \otimes \mathbb{R})$ and $\mathrm{BMO}_{\delta, \delta}(\mathbb{R} \otimes \mathbb{R})$. They differ only in which of the dyadic grids $\mathcal{D}$ and $\mathcal{D}^{\delta}$ is used in each variable. First, $f \in L_{\text {loc }}^{1}(\mathbb{R} \otimes \mathbb{R})$ belongs 
to $\mathrm{BMO}_{d, d}(\mathbb{R} \otimes \mathbb{R})$ if there is a positive constant $C$ such that for each open set $\Omega \subset \mathbb{R} \otimes \mathbb{R}$ with finite measure, the inequality

$$
\sum_{R=I \times J \in \mathcal{D} \times \mathcal{D}, R \subset \Omega}\left(f, h_{R}\right)^{2} \leq C|\Omega|
$$

holds, where $h_{R}=h_{I} \times h_{J}$, and $h_{I}$ and $h_{J}$ are the Haar functions on the intervals $I, J \in \mathcal{D}$.

Define $\mathrm{BMO}_{d, \delta}(\mathbb{R} \otimes \mathbb{R})$ in the same way, summing over $R=I \times J \in \mathcal{D} \times \mathcal{D}^{\delta}$, and similarly for $\mathrm{BMO}_{\delta, d}(\mathbb{R} \otimes \mathbb{R})$ and $\mathrm{BMO}_{\delta, \delta}(\mathbb{R} \otimes \mathbb{R})$.

We are ready to prove the main result of this section.

Theorem 3.6. Suppose $\delta \in \mathbb{R}$ is far from dyadic rationals, in the sense of condition (1.2). Then

$\mathrm{BMO}(\mathbb{R} \otimes \mathbb{R})=\mathrm{BMO}_{d, d}(\mathbb{R} \otimes \mathbb{R}) \cap \mathrm{BMO}_{d, \delta}(\mathbb{R} \otimes \mathbb{R}) \cap \mathrm{BMO}_{\delta, d}(\mathbb{R} \otimes \mathbb{R}) \cap \mathrm{BMO}_{\delta, \delta}(\mathbb{R} \otimes \mathbb{R})$,

with equivalent norms. The norm $\|\cdot\|_{\mathrm{BMO}(\mathbb{R} \otimes \mathbb{R})}$ is comparable to the maximum of the four dyadic BMO norms.

Proof. We first note that

$\mathrm{BMO}(\mathbb{R} \otimes \mathbb{R}) \subset \mathrm{BMO}_{d, d}(\mathbb{R} \otimes \mathbb{R}) \cap \mathrm{BMO}_{d, \delta}(\mathbb{R} \otimes \mathbb{R}) \cap \mathrm{BMO}_{\delta, d}(\mathbb{R} \otimes \mathbb{R}) \cap \mathrm{BMO}_{\delta, \delta}(\mathbb{R} \otimes \mathbb{R})$.

This inclusion is not trivial in the multiparameter setting. A proof (for biparameter BMO) was given in the Ph. D. thesis [22] of J. Pipher, but the best proof of this result is in S. Treil's paper [27]. There he shows that $H^{1}(\mathbb{R} \otimes \mathbb{R}) \supset H_{d, d}^{1}(\mathbb{R} \otimes \mathbb{R})$ via the characterization of these $H^{1}$ spaces in terms of the square function and the fact that the multiparameter square function acts iteratively when viewed as a vector-valued operator. Using the fact that the dual of $H^{1}(\mathbb{R} \otimes \mathbb{R})$ is $\operatorname{BMO}(\mathbb{R} \otimes \mathbb{R})$, by [5], and likewise the dual of $H_{d, d}^{1}(\mathbb{R} \otimes \mathbb{R})$ is $\mathrm{BMO}_{d, d}(\mathbb{R} \otimes \mathbb{R})$, by [1], it follows that $\mathrm{BMO}(\mathbb{R} \otimes \mathbb{R}) \subset \mathrm{BMO}_{d, d}(\mathbb{R} \otimes \mathbb{R})$.

The same argument shows that $\mathrm{BMO}(\mathbb{R} \otimes \mathbb{R})$ is contained in each of $\mathrm{BMO}_{d, \delta}(\mathbb{R} \otimes$ $\mathbb{R}), \mathrm{BMO}_{\delta, d}(\mathbb{R} \otimes \mathbb{R})$, and $\mathrm{BMO}_{\delta, \delta}(\mathbb{R} \otimes \mathbb{R})$.

Now suppose that $f \in \mathrm{BMO}_{d, d}(\mathbb{R} \otimes \mathbb{R}) \cap \mathrm{BMO}_{d, \delta}(\mathbb{R} \otimes \mathbb{R}) \cap \mathrm{BMO}_{\delta, d}(\mathbb{R} \otimes \mathbb{R}) \cap$ $\mathrm{BMO}_{\delta, \delta}(\mathbb{R} \otimes \mathbb{R})$. We must show that there is a positive constant $C$ such that the inequality

$$
\iint_{T(\Omega)}\left|f * \psi_{y_{1}} \psi_{y_{2}}\left(t_{1}, t_{2}\right)\right|^{2} \frac{d t_{1} d y_{1} d t_{2} d y_{2}}{y_{1} y_{2}} \leq C|\Omega|
$$

holds for all open sets $\Omega \subset \mathbb{R} \otimes \mathbb{R}$ with finite measure.

Fix such a set $\Omega$. For each point $\left(t_{1}, y_{1}, t_{2}, y_{2}\right)$ in the Carleson tent $T(\Omega)$, by definition the two intervals $I_{t_{1}, y_{1}}$ and $I_{t_{2}, y_{2}}$ satisfy $I_{t_{1}, y_{2}} \times I_{t_{2}, y_{2}} \subset \Omega$. By Proposition 2.1, for such $\left(t_{1}, y_{1}\right)$, we may choose an interval $I_{t_{1}, y_{1}}^{*}$ such that $I_{t_{1}, y_{1}} \subset$ $I_{t_{1}, y_{1}}^{*},\left|I_{t_{1}, y_{1}}^{*}\right| \leq C(\delta)\left|I_{t_{1}, y_{1}}\right|$, and either $I_{t_{1}, y_{1}}^{*} \in \mathcal{D}$ or $I_{t_{1}, y_{1}}^{*} \in \mathcal{D}^{\delta}$. Similarly, for 
such $\left(t_{2}, y_{2}\right)$, we may choose an interval $I_{t_{2}, y_{2}}^{*}$ such that $I_{t_{2}, y_{2}} \subset I_{t_{2}, y_{2}}^{*},\left|I_{t_{2}, y_{2}}^{*}\right| \leq$ $C(\delta)\left|I_{t_{2}, y_{2}}\right|$, and either $I_{t_{2}, y_{2}}^{*} \in \mathcal{D}$ or $I_{t_{2}, y_{2}}^{*} \in \mathcal{D}^{\delta}$. Now, we let

$$
\begin{aligned}
& \mathcal{F}_{1}:=\left\{\left(t_{1}, y_{1}, t_{2}, y_{2}\right) \in T(\Omega) \mid I_{t_{1}, y_{1}}^{*} \in \mathcal{D}, \quad I_{t_{2}, y_{2}}^{*} \in \mathcal{D}\right\} ; \\
& \mathcal{F}_{2}:=\left\{\left(t_{1}, y_{1}, t_{2}, y_{2}\right) \in T(\Omega) \mid I_{t_{1}, y_{1}}^{*} \in \mathcal{D}, \quad I_{t_{2}, y_{2}}^{*} \in \mathcal{D}^{\delta}\right\} ; \\
& \mathcal{F}_{3}:=\left\{\left(t_{1}, y_{1}, t_{2}, y_{2}\right) \in T(\Omega) \mid I_{t_{1}, y_{1}}^{*} \in \mathcal{D}^{\delta}, \quad I_{t_{2}, y_{2}}^{*} \in \mathcal{D}\right\} ; \\
& \mathcal{F}_{4}:=\left\{\left(t_{1}, y_{1}, t_{2}, y_{2}\right) \in T(\Omega) \mid I_{t_{1}, y_{1}}^{*} \in \mathcal{D}^{\delta}, \quad I_{t_{2}, y_{2}}^{*} \in \mathcal{D}^{\delta}\right\} .
\end{aligned}
$$

Then $T(\Omega)=\mathcal{F}_{1} \cup \mathcal{F}_{2} \cup \mathcal{F}_{3} \cup \mathcal{F}_{4}$, and the sets $\mathcal{F}_{i}(i=1,2,3,4)$ are pairwise disjoint. Hence

$$
\begin{aligned}
& \iint_{T(\Omega)}\left|f * \psi_{y_{1}} \psi_{y_{2}}\left(t_{1}, t_{2}\right)\right|^{2} \frac{d t_{1} d y_{1} d t_{2} d y_{2}}{y_{1} y_{2}} \\
& \quad=\sum_{i=1}^{4} \iint_{\mathcal{F}_{i}}\left|f * \psi_{y_{1}} \psi_{y_{2}}\left(t_{1}, t_{2}\right)\right|^{2} \frac{d t_{1} d y_{1} d t_{2} d y_{2}}{y_{1} y_{2}}=: G_{1}+G_{2}+G_{3}+G_{4} .
\end{aligned}
$$

We first estimate $G_{1}$. For every $\left(t_{1}, y_{1}, t_{2}, y_{2}\right)$ in $\mathcal{F}_{1}$, we have $I_{t_{1}, y_{1}}^{*} \in \mathcal{D}$ and $I_{t_{2}, y_{2}}^{*} \in \mathcal{D}$. Let $\widetilde{I}_{t_{1}, y_{1}}^{*}$ and $\widetilde{I}_{t_{2}, y_{2}}^{*}$ be the dyadic parents of $I_{t_{1}, y_{1}}^{*}$ and $I_{t_{2}, y_{2}}^{*}$, respectively. Define

$$
\widetilde{\Omega}_{1}:=\bigcup_{\left(t_{1}, y_{1}, t_{2}, y_{2}\right) \in \mathcal{F}_{1}} \widetilde{I}_{t_{1}, y_{1}}^{*} \times \widetilde{I}_{t_{2}, y_{2}}^{*} .
$$

Then

$$
\left|\widetilde{\Omega}_{1}\right|=\left|\bigcup_{\left(t_{1}, y_{1}, t_{2}, y_{2}\right) \in \mathcal{F}_{1}} \widetilde{I}_{t_{1}, y_{1}}^{*} \times \widetilde{I}_{t_{2}, y_{2}}^{*}\right| \leq\left|\left\{M_{s} \chi_{\Omega}>\frac{1}{2^{2} C(\delta)^{2}}\right\}\right| \lesssim C(\delta)^{2}|\Omega|,
$$

where $M_{s}$ is the strong maximal operator. Next, using the biparameter Haar expansion, we have

$$
f * \psi_{y_{1}} \psi_{y_{2}}\left(t_{1}, t_{2}\right)=\sum_{R=I_{1} \times I_{2} \in \mathcal{D} \times \mathcal{D}}\left(f, h_{R}\right) h_{I_{1}} * \psi_{y_{1}}\left(t_{1}\right) h_{I_{2}} * \psi_{y_{2}}\left(t_{2}\right)
$$

for every $\left(t_{1}, y_{1}, t_{2}, y_{2}\right) \in \mathcal{F}_{1}$. We now claim that: If $I_{1} \nsubseteq \widetilde{I}_{t_{1}, y_{1}}^{*}$, then $h_{I_{1}} * \psi_{y_{1}}\left(t_{1}\right)=0$.

In fact, this claim follows from the analogous estimates in the one-parameter case; see the estimates of $g_{12}$ in the proof of Theorem 3.5. More precisely, we explain it as follows. First, from the properties of $h_{I_{1}}$ and $\psi_{y_{1}}$, we see that if $I_{1} \cap \widetilde{I}_{t_{1}, y_{1}}^{*}=\emptyset$, then $h_{I_{1}} * \psi_{y_{1}}\left(t_{1}\right)=0$. Moreover, if $I_{1} \cap \widetilde{I}_{t_{1}, y_{1}}^{*} \neq \emptyset$ and $I_{1} \nsubseteq \widetilde{I}_{t_{1}, y_{1}}^{*}$, then $I_{1}$ must be larger than $\widetilde{I}_{t_{1}, y_{1}}^{*}$ since both $I_{1}$ and $\widetilde{I}_{t_{1}, y_{1}}^{*}$ are dyadic, which means that $I_{1}$ is some ancestor of $\widetilde{I}_{t_{1}, y_{1}}^{*}$. In this case, since $\psi_{y_{1}}\left(t_{1}-\cdot\right)$ is supported in $I_{t_{1}, y_{1}}$ and $h_{I_{1}}$ is constant on $I_{t_{1}, y_{1}}$, we have $h_{I_{1}} * \psi_{y_{1}}\left(t_{1}\right)=0$. Combining the two cases, we see that the claim holds.

Similarly, if $I_{2} \nsubseteq \widetilde{I}_{t_{2}, y_{2}}^{*}$, then $h_{I_{2}} * \psi_{y_{2}}\left(t_{2}\right)=0$. 
As a consequence, we have

$$
\begin{aligned}
f * \psi_{y_{1}} \psi_{y_{2}}\left(t_{1}, t_{2}\right) & =\sum_{R=I_{1} \times I_{2} \in \mathcal{D} \times \mathcal{D}}\left(f, h_{R}\right) h_{I_{1}} * \psi_{y_{1}}\left(t_{1}\right) h_{I_{2}} * \psi_{y_{2}}\left(t_{2}\right) \\
= & \sum_{R=I_{1} \times I_{2} \in \mathcal{D} \times \mathcal{D}, R \subset \widetilde{I}_{t_{1}, y_{1}}^{*} \times \widetilde{I}_{t_{2}, y_{2}}^{*}}\left(f, h_{R}\right) h_{I_{1}} * \psi_{y_{1}}\left(t_{1}\right) h_{I_{2}} * \psi_{y_{2}}\left(t_{2}\right) .
\end{aligned}
$$

We now estimate $G_{1}$. First let $P_{\widetilde{\Omega}_{1}} f$ denote the projection

$$
P_{\widetilde{\Omega}_{1}} f:=\sum_{R=I_{1} \times I_{2} \subset \widetilde{\Omega}_{1}}\left(f, h_{R}\right) h_{R} .
$$

From the results above, we have

$$
\begin{aligned}
& G_{1}:=\iint_{\mathcal{F}_{1}}\left|f * \psi_{y_{1}} \psi_{y_{2}}\left(t_{1}, t_{2}\right)\right|^{2} \frac{d t_{1} d y_{1} d t_{2} d y_{2}}{y_{1} y_{2}} \\
&=\iint_{\mathcal{F}_{1}}\left|\sum_{\substack{R=I_{1} \times I_{2} \in \mathcal{D} \times \mathcal{D}, R \subset \widetilde{I}_{t_{1}, y_{1}}^{*} \times \widetilde{I}_{t_{2}, y_{2}}^{*}}}\left(f, h_{R}\right) h_{I_{1}} * \psi_{y_{1}}\left(t_{1}\right) h_{I_{2}} * \psi_{y_{2}}\left(t_{2}\right)\right|^{2} \frac{d t_{1} d y_{1} d t_{2} d y_{2}}{y_{1} y_{2}} \\
&=\iint_{\mathcal{F}_{1}}\left|\sum_{R=I_{1} \times I_{2} \in \mathcal{D} \times \mathcal{D}, R \subset \widetilde{\Omega}_{1}}\left(f, h_{R}\right) h_{I_{1}} * \psi_{y_{1}}\left(t_{1}\right) h_{I_{2}} * \psi_{y_{2}}\left(t_{2}\right)\right|^{2} \frac{d t_{1} d y_{1} d t_{2} d y_{2}}{y_{1} y_{2}} \\
&=\iint_{\mathcal{F}_{1}}\left|P_{\widetilde{\Omega}_{1}} f * \psi_{y_{1}}\left(t_{1}\right) \psi_{y_{2}}\left(t_{2}\right)\right|^{2} \frac{d t_{1} d y_{1} d t_{2} d y_{2}}{y_{1} y_{2}},
\end{aligned}
$$

The last equality holds since the terms $R \subset \widetilde{\Omega}_{1}$ but $R \not \subset \widetilde{I}_{t_{1}, y_{1}}^{*} \times \widetilde{I}_{t_{2}, y_{2}}^{*}$ are zero.

Then, using the $L^{2}$ boundedness of the Littlewood-Paley $g$-function, we see that

$$
\begin{aligned}
G_{1} & \leq \iint_{\mathbb{R}_{+}^{2} \times \mathbb{R}_{+}^{2}}\left|P_{\widetilde{\Omega}_{1}} f * \psi_{y_{1}}\left(t_{1}\right) \psi_{y_{2}}\left(t_{2}\right)\right|^{2} \frac{d t_{1} d y_{1} d t_{2} d y_{2}}{y_{1} y_{2}} \\
& \leq C\left\|P_{\widetilde{\Omega}_{1}} f\right\|_{L^{2}(\mathbb{R} \otimes \mathbb{R})}^{2}=C \sum_{R \in \mathcal{D} \times \mathcal{D}}\left(P_{\widetilde{\Omega}_{1}} f, h_{R}\right)^{2}=C \sum_{R \subset \widetilde{\Omega}_{1}}\left(f, h_{R}\right)^{2} \\
& \leq C\left|\widetilde{\Omega}_{1}\right|\|f\|_{\mathrm{BMO}_{d, d}(\mathbb{R} \otimes \mathbb{R})}^{2} \leq 4 C \cdot C(\delta)^{2}|\Omega|\|f\|_{\mathrm{BMO}_{d, d}(\mathbb{R} \otimes \mathbb{R})}^{2} .
\end{aligned}
$$

Repeating the proof above, we find that $G_{2} \leq 4 C \cdot C(\delta)^{2}|\Omega|\|f\|_{\mathrm{BMO}_{d, \delta}(\mathbb{R} \otimes \mathbb{R})}^{2}$, $G_{3} \leq 4 C \cdot C(\delta)^{2}|\Omega|\|f\|_{\mathrm{BMO}_{\delta, d}(\mathbb{R} \otimes \mathbb{R})}^{2}$ and $G_{4} \leq 4 C \cdot C(\delta)^{2}|\Omega|\|f\|_{\mathrm{BMO}_{\delta, \delta}(\mathbb{R} \otimes \mathbb{R})}^{2}$. Combining the estimates for $G_{1}, \ldots, G_{4}$, we see that inequality (3.12) holds with a constant $C$ independent of $\Omega$, as required. In particular, $f \in \operatorname{BMO}(\mathbb{R} \otimes \mathbb{R})$ and

$$
\begin{aligned}
& \|f\|_{\mathrm{BMO}(\mathbb{R} \otimes \mathbb{R})} \leq C \cdot C(\delta) \\
& \quad \times \max \left\{\|f\|_{\mathrm{BMO}_{d, d}(\mathbb{R} \otimes \mathbb{R})},\|f\|_{\mathrm{BMO}_{d, \delta}(\mathbb{R} \otimes \mathbb{R})},\|f\|_{\mathrm{BMO}_{\delta, d}(\mathbb{R} \otimes \mathbb{R})},\|f\|_{\mathrm{BMO}_{\delta, \delta}(\mathbb{R} \otimes \mathbb{R})}\right\},
\end{aligned}
$$

where $C$ depends only on the $C_{\psi}$ in condition (3.1). 


\section{VMO and product VMO}

We begin with the one-parameter case. We state three definitions of $\operatorname{VMO}(\mathbb{R})$ and show that they are equivalent. The space VMO of functions of vanishing mean oscillation on the circle $\mathbb{T}$ was introduced by Sarason in [25] as the set of integrable functions on $\mathbb{T}$ satisfying $\lim _{\delta \rightarrow 0} \sup _{I:|I| \leq \delta} f_{I}\left|f-f_{I}\right| d x=0$. This space is the closure in the $\mathrm{BMO}$ norm of the subspace of $\mathrm{BMO}(\mathbb{T})$ consisting of all uniformly continuous functions on $\mathbb{T}$.

The analogous space $\operatorname{VMO}(\mathbb{R})$ on the real line was defined by Coifman and Weiss [7], where they proved that it is the predual of the Hardy space $H^{1}(\mathbb{R})$.

Definition $4.1([7])$. $\operatorname{VMO}(\mathbb{R})$ is the closure of $C_{0}^{\infty}(\mathbb{R})$ in the $\operatorname{BMO}(\mathbb{R})$ norm from (3.5).

The second definition is in terms of oscillation conditions.

Definition 4.2. The space $\operatorname{VMO}(\mathbb{R})$ is the set of all functions $f \in \operatorname{BMO}(\mathbb{R})$ satisfying the following conditions:

(a) $\lim _{\delta \rightarrow 0} \sup _{Q:|Q|<\delta} f_{Q}\left|f-f_{Q}\right| d x=0$;

(b) $\lim _{N \rightarrow \infty} \sup _{Q:|Q|>N} f_{Q}\left|f-f_{Q}\right| d x=0$; and

(c) $\lim _{R \rightarrow \infty} \sup _{Q: Q \cap B(0, R)=\emptyset} f_{Q}\left|f-f_{Q}\right| d x=0$,

where $Q$ denotes an interval in $\mathbb{R}$.

The third definition is in terms of Carleson measures.

Definition 4.3. A function $f \in \operatorname{BMO}(\mathbb{R})$ belongs to $\operatorname{VMO}(\mathbb{R})$ if

(a) $\lim _{\delta \rightarrow 0} \sup _{Q:|Q|<\delta} \frac{1}{|Q|} \int_{T(Q)}\left|f * \psi_{y}(t)\right|^{2} \frac{d t d y}{y}=0$;

(b) $\lim _{N \rightarrow \infty} \sup _{Q:|Q|>N} \frac{1}{|Q|} \int_{T(Q)}\left|f * \psi_{y}(t)\right|^{2} \frac{d t d y}{y}=0$; and

(c) $\lim _{R \rightarrow \infty} \sup _{Q: Q \cap B(0, R)=\emptyset} \frac{1}{|Q|} \int_{T(Q)}\left|f * \psi_{y}(t)\right|^{2} \frac{d t d y}{y}=0$,

where $\psi$ is any function of the form specified in Proposition 3.1.

Theorem 4.4. Definitions 4.1, 4.2, and 4.3 of $\operatorname{VMO}(\mathbb{R})$ are equivalent.

Proof. For the proof of the equivalence of Definitions 4.1 and 4.2, see the Lemma in Section 3 of [28], pp. 166-167. See also Theorem 7 of [3]. 
The equivalence of Definitions 4.2 and 4.3 can be shown as follows. First, it is a routine estimate that

$$
\frac{1}{|Q|} \int_{T(Q)}\left|\psi_{y} * f(t)\right|^{2} \frac{d t d y}{y} \leq C f_{4 Q}\left|f(x)-f_{4 Q}\right|^{2} d x
$$

where $Q$ is an arbitrary interval in $\mathbb{R}, 4 Q$ is the interval with the same midpoint as $Q$ and four times the length, and $C$ is a constant independent of $Q$ and $f$. As a consequence, (a), (b) and (c) in Definition 4.3 follow directly from (a), (b) and (c) in Definition 4.2. Conversely, suppose $f$ satisfies Definition 4.3. Then it follows from Proposition 3.3 in [9] that $f$ satisfies Definition 4.2. We note that [9] deals with the generalized space $\mathrm{VMO}_{L}\left(\mathbb{R}^{n}\right)$ of $\mathrm{VMO}$ functions associated to a differential operator $L$ satisfying the conditions that $L$ has a bounded holomorphic functional calculus on $L^{2}\left(\mathbb{R}^{n}\right)$ and that the heat kernel of the analytic semigroup generated by $L$ has suitable upper bounds. We need only the special case when $L$ is the Laplacian $\Delta$. It is shown in Proposition 3.6 in [9], by an argument using the tent space corresponding to $\mathrm{VMO}$, that $\mathrm{VMO}_{\Delta}\left(\mathbb{R}^{n}\right)$ coincides with the usual VMO as in Definition 4.2.

We turn to the dyadic one-parameter case. Again we give three equivalent definitions. First, $\mathrm{VMO}_{d}(\mathbb{R})$ is the closure of the space $C_{0}^{\infty}(\mathbb{R})$ in the dyadic $\mathrm{BMO}_{d}(\mathbb{R})$ norm of formula (3.3). Second, in terms of averages, we define $\mathrm{VMO}_{d}(\mathbb{R})$ as in Definition 4.2 of $\mathrm{VMO}(\mathbb{R})$ but taking the three suprema over only dyadic intervals $I$ instead of arbitrary intervals $Q$. The third definition is in terms of a Carleson condition on Haar coefficients, as follows.

Definition 4.5. A function $f \in \mathrm{BMO}_{d}(\mathbb{R})$ belongs to the dyadic VMO space $\mathrm{VMO}_{d}(\mathbb{R})$ if
(a) $\lim _{\delta \rightarrow 0} \sup _{J: J \in \mathcal{D}} \frac{1}{|J|} \sum_{I: I \subset J, I \in \mathcal{D},|I|<\delta}\left(f, h_{I}\right)^{2}=0 ;$
(b) $\lim _{N \rightarrow \infty} \sup _{J: J \in \mathcal{D}} \frac{1}{|J|} \sum_{I: I \subset J, I \in \mathcal{D},|I|>N}\left(f, h_{I}\right)^{2}=0$; and
(c) $\lim _{R \rightarrow \infty} \sup _{J: J \in \mathcal{D}} \frac{1}{|J|} \sum_{I: I \subset J, I \in \mathcal{D}, I \cap B(0, R)=\emptyset}\left(f, h_{I}\right)^{2}=0$.

Just as for dyadic BMO (Definition 3.3), allowing $J$ in Definition 4.5 to range over all intervals, not just dyadic intervals, yields the same space $\mathrm{VMO}_{d}(\mathbb{R})$ with a comparable norm.

Theorem 4.6. The following definitions of the dyadic $\mathrm{VMO}$ space $\mathrm{VMO}_{d}(\mathbb{R})$ are equivalent:

(1) The definition of $\mathrm{VMO}_{d}(\mathbb{R})$ as the closure of $C_{0}^{\infty}(\mathbb{R})$ in the $\mathrm{BMO}_{d}(\mathbb{R})$ norm (3.3).

(2) The dyadic version of Definition 4.2, in terms of oscillations. 
(3) Definition 4.5, in terms of Haar coefficients.

Similarly, for each $\delta \in \mathbb{R}$, the three analogous definitions for the dyadic VMO space $\mathrm{VMO}_{\delta}(\mathbb{R})$ defined with respect to $\mathcal{D}^{\delta}$ are equivalent.

Proof. The proof of the equivalence of definitions (1) and (2) follows fom the corresponding proof in the continuous case. The equivalence of definitions (2) and (3) is proved in the same way as the equivalence of Definitions 3.2 and 3.3 of $\mathrm{BMO}_{d}(\mathbb{R})$.

Next we consider the product VMO space $\operatorname{VMO}(\mathbb{R} \otimes \mathbb{R})$, which was first defined in [17]. Here we give only two definitions, since the one-parameter definition in terms of oscillations does not generalize naturally. First, $\operatorname{VMO}(\mathbb{R} \otimes \mathbb{R})$ is the closure of $C_{0}^{\infty}(\mathbb{R} \otimes \mathbb{R})$ in the product $\mathrm{BMO}(\mathbb{R} \otimes \mathbb{R})$ norm. The second definition is in terms of Carleson measures, as follows.

Definition 4.7. A function $f \in \operatorname{BMO}(\mathbb{R} \otimes \mathbb{R})$ belongs to $\operatorname{VMO}(\mathbb{R} \otimes \mathbb{R})$ if

(a) $\lim _{\delta \rightarrow 0} \sup _{\Omega} \frac{1}{|\Omega|} \sum_{R \in \mathcal{D} \times \mathcal{D}: R \subset \Omega,|R|<\delta} \int_{T(R)}\left|f * \psi_{y}(t)\right|^{2} \frac{d t_{1} d y_{1} d t_{2} d y_{2}}{y_{1} y_{2}}=0$;

(b) $\lim _{N \rightarrow \infty} \sup _{\Omega} \frac{1}{|\Omega|} \sum_{R \in \mathcal{D} \times \mathcal{D}: R \subset \Omega, \operatorname{diam}(R)>N} \int_{T(R)}\left|f * \psi_{y}(t)\right|^{2} \frac{d t_{1} d y_{1} d t_{2} d y_{2}}{y_{1} y_{2}}=0$; and

(c) $\lim _{R \rightarrow \infty} \sup _{\Omega} \frac{1}{|\Omega|} \sum_{R \in \mathcal{D} \times \mathcal{D}: R \subset \Omega, R \not \subset B(0, N)} \int_{T(R)}\left|f * \psi_{y}(t)\right|^{2} \frac{d t_{1} d y_{1} d t_{2} d y_{2}}{y_{1} y_{2}}=0$.

Here and in the definitions below, $\Omega$ ranges over all open sets in $\mathbb{R} \otimes \mathbb{R}$ of finite measure.

Theorem 4.8. The following definitions of product $\operatorname{VMO}(\mathbb{R} \otimes \mathbb{R})$ are equivalent:

(1) $\operatorname{VMO}(\mathbb{R} \otimes \mathbb{R})$ as the closure of $C_{0}^{\infty}(\mathbb{R} \otimes \mathbb{R})$ in the $\mathrm{BMO}(\mathbb{R} \otimes \mathbb{R})$ norm.

(2) Definition 4.7, in terms of Carleson measures.

Proof. A short calculation shows that Definition 4.7 is equivalent to the definition of $\operatorname{VMO}(\mathbb{R} \otimes \mathbb{R})$ given in Proposition 5.1(ii) of [17]. In [17] the equivalence of this last definition and the definition in terms of $C_{0}^{\infty}(\mathbb{R} \otimes \mathbb{R})$ is proved.

Finally, we define the dyadic product $\mathrm{VMO}$ space $\mathrm{VMO}_{d, d}(\mathbb{R} \otimes \mathbb{R})$, in two ways. We have not found this space in the literature. First, $\mathrm{VMO}_{d, d}(\mathbb{R} \otimes \mathbb{R})$ is the closure of $C_{0}^{\infty}(\mathbb{R} \otimes \mathbb{R})$ in the dyadic $\mathrm{BMO}_{d, d}(\mathbb{R} \otimes \mathbb{R})$ norm. The second definition is in terms of a Carleson condition on the Haar coefficients, as follows.

Definition 4.9. A function $f \in \mathrm{BMO}_{d, d}(\mathbb{R} \otimes \mathbb{R})$ belongs to the dyadic product $\mathrm{VMO}$ space $\mathrm{VMO}_{d, d}(\mathbb{R} \otimes \mathbb{R})$ if
(a) $\lim _{\delta \rightarrow 0} \sup _{\Omega} \frac{1}{|\Omega|} \sum_{R \in \mathcal{D} \times \mathcal{D}: R \subset \Omega,|R|<\delta}\left(f, h_{R}\right)^{2}=0$; 
(b) $\lim _{N \rightarrow \infty} \sup _{\Omega} \frac{1}{|\Omega|} \sum_{R \in \mathcal{D} \times \mathcal{D}: R \subset \Omega, \operatorname{diam}(R)>N}\left(f, h_{R}\right)^{2}=0$; and

(c) $\lim _{N \rightarrow \infty} \sup _{\Omega} \frac{1}{|\Omega|} \sum_{R \in \mathcal{D} \times \mathcal{D}: R \subset \Omega, R \not \subset B(0, N)}\left(f, h_{R}\right)^{2}=0$.

We define $\mathrm{VMO}_{d, \delta}(\mathbb{R} \otimes \mathbb{R}), \mathrm{VMO}_{\delta, d}(\mathbb{R} \otimes \mathbb{R})$, and $\mathrm{VMO}_{\delta, \delta}(\mathbb{R} \otimes \mathbb{R})$ similarly.

Theorem 4.10. The following definitions of dyadic product $\mathrm{VMO}_{d, d}(\mathbb{R} \otimes \mathbb{R})$ are equivalent:

(1) The definition of $\mathrm{VMO}_{d, d}(\mathbb{R} \otimes \mathbb{R})$ as the closure, in the $\mathrm{BMO}_{d, d}(\mathbb{R} \otimes \mathbb{R})$ norm, of $C_{0}^{\infty}(\mathbb{R} \otimes \mathbb{R})$.

(2) Definition 4.9, in terms of a Carleson condition on the Haar coefficients.

The analogous results hold for the spaces $\mathrm{VMO}_{d, \delta}(\mathbb{R} \otimes \mathbb{R}), \mathrm{VMO}_{\delta, d}(\mathbb{R} \otimes \mathbb{R})$, and $\mathrm{VMO}_{\delta, \delta}(\mathbb{R} \otimes \mathbb{R})$.

Proof. We follow the ideas given in [17] for the continuous case. Denote by FH the linear space of finite linear combinations of the Haar basis $\left\{h_{R}: R \in \mathcal{D} \times \mathcal{D}\right\}$. We first claim that

$$
\operatorname{clos}_{\mathrm{BMO}_{d, d}} \mathrm{FH}=\mathrm{VMO}_{d, d} .
$$

In fact, from Definition 4.9, it is immediate that every Haar function $h_{R}$ belongs to $\mathrm{VMO}_{d, d}$. Conversely, for each $f \in \mathrm{VMO}_{d, d}$, set

$$
f_{n}:=\sum_{R \in \mathcal{D} \times \mathcal{D}:} \sum_{R \subset B\left(0,2^{n}\right), 2^{-n} \leq|R| \leq 2^{n}}\left(f, h_{R}\right) h_{R}
$$

for every positive integer $n$. Then it is clear that $f_{n} \in \mathrm{FH}$ for each $n$. Moreover, $\left\|f-f_{n}\right\|_{\mathrm{BMO}_{d, d}}$ goes to 0 as $n$ tends to infinity, by conditions (a), (b) and (c) in Definition 4.9. Hence the claim holds.

Next, we claim that

$$
\operatorname{clos}_{\mathrm{BMO}_{d, d}} C_{0}^{\infty}=\operatorname{clos}_{\mathrm{BMO}_{d, d}} \mathrm{FH} .
$$

In fact, we can see that $C_{0}^{\infty} \subset \operatorname{clos}_{\mathrm{BMO}_{d, d}} \mathrm{FH}$ since for every $\varphi \in C_{0}^{\infty}$, we find that $\varphi$ satisfies conditions (a), (b) and (c) in Definition 4.9. Hence by taking $\varphi_{n}$ as in equation (4.1), we can approximate $\varphi$ by functions in FH. Conversely, it is easy to verify that $\operatorname{clos}_{\mathrm{BMO}_{d, d}} C_{0}^{\infty} \supset \mathrm{FH}$.

The proof of Theorem 4.10 is complete.

Theorem 4.11. Suppose $\delta \in \mathbb{R}$ is far from dyadic rationals, in the sense of condition (1.2). Then in the one-parameter case,

$$
\operatorname{VMO}(\mathbb{R})=\mathrm{VMO}_{d}(\mathbb{R}) \cap \operatorname{VMO}_{\delta}(\mathbb{R}),
$$

and in the biparameter case,

$\mathrm{VMO}(\mathbb{R} \otimes \mathbb{R})=\mathrm{VMO}_{d, d}(\mathbb{R} \otimes \mathbb{R}) \cap \mathrm{VMO}_{d, \delta}(\mathbb{R} \otimes \mathbb{R}) \cap \mathrm{VMO}_{\delta, d}(\mathbb{R} \otimes \mathbb{R}) \cap \mathrm{VMO}_{\delta, \delta}(\mathbb{R} \otimes \mathbb{R})$. 
Proof. The inclusion $\mathrm{VMO}(\mathbb{R}) \subset \mathrm{VMO}_{d}(\mathbb{R}) \cap \mathrm{VMO}_{\delta}(\mathbb{R})$ follows directly from the definitions of $\operatorname{VMO}(\mathbb{R}), \mathrm{VMO}_{d}(\mathbb{R})$, and $\mathrm{VMO}_{\delta}(\mathbb{R})$ in terms of oscillations.

The proof of the other inclusion $\mathrm{VMO}(\mathbb{R}) \supset \mathrm{VMO}_{d}(\mathbb{R}) \cap \mathrm{VMO}_{\delta}(\mathbb{R})$ involves only minor modifications of our proof for $\mathrm{BMO}(\mathbb{R})$ above. We use the definition of $\mathrm{VMO}_{d}(\mathbb{R})$ and $\mathrm{VMO}_{\delta}(\mathbb{R})$ in terms of Haar coefficients (Definition 4.5). The key point is that the constant $C$ in inequality (3.9) is replaced by the $\varepsilon$ from Definition 4.5. We omit the details.

For the case of product VMO, we first show that $\operatorname{VMO}(\mathbb{R} \otimes \mathbb{R}) \subset \mathrm{VMO}_{d, d}(\mathbb{R} \otimes \mathbb{R})$. Take $f \in \operatorname{VMO}(\mathbb{R} \otimes \mathbb{R})$. Then $f$ is the limit in the $\mathrm{BMO}(\mathbb{R} \otimes \mathbb{R})$ norm of a sequence of functions $f_{n}$ in $C_{0}^{\infty}(\mathbb{R} \otimes \mathbb{R})$. Then $\left\{f_{n}\right\} \subset \mathrm{BMO}(\mathbb{R} \otimes \mathbb{R}) \subset \mathrm{BMO}_{d, d}(\mathbb{R} \otimes \mathbb{R})$, and also $f_{n}$ converges to $f$ in the $\mathrm{BMO}_{d}(\mathbb{R} \otimes \mathbb{R})$ norm. Therefore $f$ belongs to $\mathrm{VMO}_{d, d}(\mathbb{R} \otimes \mathbb{R})$, as required. The same argument shows that $f$ belongs to each of $\mathrm{VMO}_{d, \delta}(\mathbb{R} \otimes \mathbb{R}), \mathrm{VMO}_{\delta, d}(\mathbb{R} \otimes \mathbb{R})$, and $\mathrm{VMO}_{\delta, \delta}(\mathbb{R} \otimes \mathbb{R})$.

Again, we can prove the other inclusion $(\supset)$ via minor modifications of our $\mathrm{BMO}(\mathbb{R} \otimes \mathbb{R})$ proof, using the definition of our dyadic product VMO spaces in terms of Haar coefficients (Definition 4.9). Again, we omit the details.

\section{Hardy spaces}

In this section we present our results on Hardy spaces. Here we use sums, not intersections, of dyadic spaces, since the dyadic Hardy spaces are subsets, not supersets, of the continuous Hardy space. We also show (Theorem 5.4) that the duality relations between VMO, $H^{1}$, and BMO allow us to deduce the $H^{1}$ and BMO structure theorems from the VMO structure theorem. We work in the biparameter case.

The one-parameter result that the Hardy space $H^{1}$ can be written as the sum of two suitable dyadic Hardy spaces $\left(H^{1}(\mathbb{R})=H_{d}^{1}(\mathbb{R})+H_{\delta}^{1}(\mathbb{R})\right.$, for $\delta$ satisfying condition (1.2)) is proved in [19].

For the multiparameter case, let $H^{1}(\mathbb{R} \otimes \mathbb{R})$ denote the product Hardy space. Chang and Fefferman [5] showed that the dual of $H^{1}(\mathbb{R} \otimes \mathbb{R})$ is the product BMO space $\mathrm{BMO}(\mathbb{R} \otimes \mathbb{R})$, as mentioned in Section 3. Lacey, Terwilleger and Wick [17] showed that the predual of $H^{1}(\mathbb{R} \otimes \mathbb{R})$ is the product $\operatorname{VMO}$ space $\operatorname{VMO}(\mathbb{R} \otimes \mathbb{R})$, as noted in Section 4.

Next, as in the proof of Theorem 3.6, we denote by $H_{d, d}^{1}(\mathbb{R} \otimes \mathbb{R})$ the dyadic product Hardy space with respect to dyadic rectangles $R \in \mathcal{D} \times \mathcal{D}$, whose norm is defined as

$$
\|f\|_{H_{d, d}^{1}(\mathbb{R} \otimes \mathbb{R})}:=\left\|S_{d, d} f\right\|_{L^{1}(\mathbb{R} \otimes \mathbb{R})}=\left\|\left(\sum_{R \in \mathcal{D} \times \mathcal{D}}\left(f, h_{R}\right)^{2}|R|^{-1} \chi_{R}\right)^{1 / 2}\right\|_{1} .
$$

Here $S_{d, d}$ denotes the dyadic square function with respect to $\mathcal{D} \times \mathcal{D}$, and $\chi_{R}$ is the characteristic function of $R$. For more information on the dyadic product Hardy space, see [27]. We define the dyadic product Hardy spaces $H_{d, \delta}^{1}(\mathbb{R} \otimes \mathbb{R})$, $H_{\delta, d}^{1}(\mathbb{R} \otimes \mathbb{R})$ and $H_{\delta, \delta}^{1}(\mathbb{R} \otimes \mathbb{R})$ similarly, in terms of the dyadic square functions $S_{d, \delta}, S_{\delta, d}$, and $S_{\delta, \delta}$ respectively. 
We note that there is an equivalent definition of dyadic product $H^{1}$ in terms of the atomic decomposition, and that the norms $\|\cdot\|_{H_{d, d}^{1}(\mathbb{R} \otimes \mathbb{R})}$ and $\|\cdot\|_{H_{d, d, \text { at }}^{1}(\mathbb{R} \otimes \mathbb{R})}$ are comparable.

Lemma 5.1. Dyadic product BMO is the dual of dyadic product $H^{1}$, and dyadic product $H^{1}$ is the dual of dyadic product VMO:

$$
\left(H_{d, d}^{1}(\mathbb{R} \otimes \mathbb{R})\right)^{*}=\mathrm{BMO}_{d, d}(\mathbb{R} \otimes \mathbb{R}), \quad\left(\mathrm{VMO}_{d, d}(\mathbb{R} \otimes \mathbb{R})\right)^{*}=H_{d, d}^{1}(\mathbb{R} \otimes \mathbb{R}),
$$

and similarly for $H_{d, \delta}^{1}, H_{\delta, d}^{1}, H_{\delta, \delta}^{1}$ and the corresponding BMO and VMO spaces.

Proof. The first proof of the duality of dyadic biparameter $H^{1}$ and BMO is in [1]. See also Theorem 4.2 of [15] for a proof in a more general setting of product sequence spaces.

The proof of the duality of dyadic product VMO and $H^{1}$ is similar to the proof of the continuous version $(\operatorname{VMO}(\mathbb{R} \otimes \mathbb{R}))^{*}=H^{1}(\mathbb{R} \otimes \mathbb{R})$ as shown in [17], where they relied on the facts that $\left(H^{1}(\mathbb{R} \otimes \mathbb{R})\right)^{*}=\mathrm{BMO}(\mathbb{R} \otimes \mathbb{R})$ and $\operatorname{clos}_{H^{1}} \mathrm{FW}=H^{1}$. Here FW means the linear space of finite linear combinations of product wavelets. Correspondingly, we have the facts that $\left(H_{d, d}^{1}(\mathbb{R} \otimes \mathbb{R})\right)^{*}=\mathrm{BMO}_{d, d}(\mathbb{R} \otimes \mathbb{R})$ and that $\operatorname{clos}_{H_{d, d}^{1}} \mathrm{FH}=H_{d, d}^{1}$, where the latter follows from the definition of the norm of $H_{d, d}^{1}(\mathbb{R} \otimes \mathbb{R})$.

Then we have the following result.

Theorem 5.2. Suppose $\delta \in \mathbb{R}$ is far from dyadic rationals, in the sense of condition (1.2). Then

$$
H^{1}(\mathbb{R} \otimes \mathbb{R})=H_{d, d}^{1}(\mathbb{R} \otimes \mathbb{R})+H_{d, \delta}^{1}(\mathbb{R} \otimes \mathbb{R})+H_{\delta, d}^{1}(\mathbb{R} \otimes \mathbb{R})+H_{\delta, \delta}^{1}(\mathbb{R} \otimes \mathbb{R}),
$$

with equivalent norms (see Definition 5.3).

Proof. The result follows from the intersection result for product VMO (Theorem 4.11), Theorem 5.4 below, and the duality of dyadic product VMO and $H^{1}$ (Lemma 5.1). One can also obtain a direct proof via the atomic decomposition.

In a different direction, we now show that the duality relations between VMO, $H^{1}$ and BMO allow us to deduce our structure theorems for $H^{1}$ and BMO from that for $\mathrm{VMO}$.

Definition 5.3. Let $Y$ be a complete normed space, with subspaces $Y_{1}$ and $Y_{2}$. We say that $Y=Y_{1}+Y_{2}$ with equivalent norms if each $g \in Y$ can be written (not necessarily uniquely) as $g=g_{1}+g_{2}$, with $g_{1} \in Y_{1}$ and $g_{2} \in Y_{2}$, and if

$$
\|g\|_{Y} \sim \inf \left\{\left\|g_{1}\right\|_{Y_{1}}+\left\|g_{2}\right\|_{Y_{2}}: g_{1}+g_{2}=g, g_{1} \in Y_{1}, g_{2} \in Y_{2}\right\} .
$$

Note that for $Y=H^{1}, Y_{1}=H_{d}^{1}$ and $Y_{2}=H_{\delta}^{1}$, the decomposition $g=g_{d}+g_{\delta}$ is not unique, since $H_{d}^{1} \cap H_{\delta}^{1} \supsetneq\{0\}$; to see that the containment is proper, consider a sufficiently small multiple of an $H^{1}$ atom whose support lies in the intersection of a dyadic interval and a $\delta$-dyadic interval.

We will use the facts that $\|\cdot\|_{H^{1}} \sim\|\cdot\|_{\mathrm{VMO}^{*}}$ and $\|\cdot\|_{\mathrm{BMO}} \sim\|\cdot\|_{\left(H^{1}\right)^{*}}$. 
Theorem 5.4. Take $\delta \in \mathbb{R}$. Consider the function spaces VMO, $H^{1}$, BMO and their dyadic analogues, defined on $\mathbb{R} \otimes \mathbb{R}$.

(i) If $\mathrm{VMO}=\mathrm{VMO}_{d, d} \cap \mathrm{VMO}_{d, \delta} \cap \mathrm{VMO}_{\delta, d} \cap \mathrm{VMO}_{\delta, \delta}$ with equivalent norms, then $H^{1}=H_{d, d}^{1}+H_{d, \delta}^{1}+H_{\delta, d}^{1}+H_{\delta, \delta}^{1}$ with equivalent norms.

(ii) If $H^{1}=H_{d, d}^{1}+H_{d, \delta}^{1}+H_{\delta, d}^{1}+H_{\delta, \delta}^{1}$ with equivalent norms, then $\mathrm{BMO}=$ $\mathrm{BMO}_{d, d} \cap \mathrm{BMO}_{d, \delta} \cap \mathrm{BMO}_{\delta, d} \cap \mathrm{BMO}_{\delta, \delta}$ with equivalent norms.

Proof. This result is an instantiation of general theorems in functional analysis. For part (i), we have Banach spaces $X, X_{1}, \ldots, X_{k}$, and their duals $Y, Y_{1}, \ldots$, $Y_{k}$. Since $X=X_{1} \cap \cdots \cap X_{k}$, it follows that $Y=Y_{1}+\cdots+Y_{k}$ with equivalent norms. Part (ii) is similar.

\section{Maximal functions}

For $f \in L_{\text {loc }}^{1}(\mathbb{R})$ let $M(f)$ denote the Hardy-Littlewood maximal function, defined by

$$
M(f)(x):=\sup _{Q \ni x} \frac{1}{|Q|} \int_{Q}|f(y)| d y,
$$

where the supremum is taken over all intervals $Q \subset \mathbb{R}$ that contain $x$. Similarly, denote by $M_{d}(f)$ (resp. $M_{\delta}(f)$ ) the dyadic (resp. $\delta$-dyadic) maximal function; here the supremum is taken over only those intervals $I \in \mathcal{D}$ (resp. $I \in \mathcal{D}^{\delta}$ ) that contain $x$.

In the multiparameter case, instead of the Hardy-Littlewood maximal function we consider the strong maximal function $M_{s}(f)$, defined as follows. For $f \in$ $L_{\text {loc }}^{1}(\mathbb{R} \otimes \mathbb{R})$, let

$$
M_{s}(f)(x, y):=\sup _{R \ni(x, y)} \frac{1}{|R|} \int_{R}|f(u, v)| d u d v
$$

where the supremum is taken over all rectangles $R \subset \mathbb{R} \otimes \mathbb{R}$ that contain $(x, y)$.

Next, let $M_{s}^{d, d}$ denote the dyadic strong maximal function, defined by restricting the supremum in formula (6.1) to dyadic rectangles $R \in \mathcal{D} \times \mathcal{D}$ that contain $(x, y)$. Similarly, define $M_{s}^{d, \delta}(f), M_{s}^{\delta, d}(f)$ and $M_{s}^{\delta, \delta}(f)$ using $\mathcal{D} \times \mathcal{D}^{\delta}, \mathcal{D}^{\delta} \times \mathcal{D}$ and $\mathcal{D}^{\delta} \times \mathcal{D}^{\delta}$ respectively.

Theorem 6.1. Suppose $\delta \in \mathbb{R}$ is far from dyadic rationals, in the sense of condition (1.2). Then the following assertions hold:

(i) For each $f \in L_{\text {loc }}^{1}(\mathbb{R}), M(f)$ is comparable to $M_{d}(f)+M_{\delta}(f)$.

(ii) For each $f \in L_{\text {loc }}^{1}(\mathbb{R} \otimes \mathbb{R}), M_{s}(f)$ is comparable to $M_{s}^{d, d}(f)+M_{s}^{d, \delta}(f)+$ $M_{s}^{\delta, d}(f)+M_{s}^{\delta, \delta}(f)$.

The implicit constants are independent of $f$. 
Proof. It is immediate from the definitions that $M_{d}(f) \leq M(f)$ and $M_{\delta}(f) \leq M(f)$ for every $f \in L_{\text {loc }}^{1}(\mathbb{R})$. Conversely, for each interval $Q \subset \mathbb{R}$, by Proposition 2.1, there is an interval $I$ such that $Q \subset I,|I| \leq C(\delta)|Q|$ and either $I \in \mathcal{D}$ or $I \in \mathcal{D}^{\delta}$. Then

$$
\frac{1}{|Q|} \int_{Q}|f(y)| d y \leq C(\delta) \frac{1}{|I|} \int_{I}|f(y)| d y,
$$

and therefore $M(f) \leq C(\delta)\left(M_{\delta}(f)+M_{d}(f)\right)$.

The multiparameter proof is similar; the point is that each rectangle in $\mathbb{R} \otimes \mathbb{R}$ is contained in a dyadic rectangle that is not much larger and that belongs to one of the four collections $\mathcal{D} \times \mathcal{D}, \mathcal{D} \times \mathcal{D}^{\delta}, \mathcal{D}^{\delta} \times \mathcal{D}$, or $\mathcal{D}^{\delta} \times \mathcal{D}^{\delta}$. We omit the details.

\section{Doubling, $A_{p}$, and $R H_{p}$ weights}

\subsection{One-parameter doubling, $A_{p}$, and $R H_{p}$ weights}

We prove that $A_{p}$ is the intersection of two suitable translates of dyadic $A_{p}$ for $1 \leq p \leq \infty$, and similarly for $R H_{p}$ with $1 \leq p \leq \infty$, and for doubling weights.

The $A_{p}$ weights were identified by Muckenhoupt [20] as the weights $\omega$ for which the Hardy-Littlewood maximal function is bounded from $L^{p}(d \mu)$ to itself for $1<$ $p<\infty$, where $d \mu=\omega(x) d x$. For $p=1$, the $A_{1}$ weights were identified as the weights $\omega$ satisfying $M(\omega)(x) \leq C \omega(x)$, a.e. $x \in \mathbb{R}$. And for $p=\infty, A_{\infty}$ is the union of $A_{p}$ for all $1 \leq p<\infty$. For an equivalent definition of $A_{p}$ in terms of an integral condition, see for example pp. 678-679, 695 of [13]. See pp. 693-694 of [13] for the definition of the reverse-Hölder weights in terms of an integral condition. See for example [11], [13] or [10] for the theory and history of $A_{p}$ weights, $1 \leq p \leq \infty$, and of $R H_{p}$ weights for $1<p<\infty$. The class $R H_{\infty}$ was defined in [8], and the class $R H_{1}$ was defined in [2] and, via an equivalent definition, in [14]. We write $A_{p}(\omega)$ and $R H_{p}(\omega)$ for the $A_{p}$ constant and the reverse-Hölder- $p$ constant of $\omega$, respectively, for $1 \leq p \leq \infty$.

The dyadic $A_{p}$ classes $A_{p}^{d}$ for $1 \leq p \leq \infty$ are defined analogously, using the dyadic Hardy-Littlewood maximal function or suitable integral conditions. For the dyadic reverse-Hölder classes it is necessary to assume the weight is dyadic doubling, in addition to satisfying an integral condition. We define $R H_{p}^{d}(\omega)$ to be the larger of the dyadic doubling constant and the constant from the integral condition. The $\delta$-dyadic classes $A_{p}^{\delta}$ and $R H_{p}^{\delta}$ are defined similarly, using $\mathcal{D}^{\delta}$ in place of $\mathcal{D}$.

It is shown in [2] that a weight $\omega$ belongs to $A_{\infty}$ if and only if $\omega$ belongs to $R H_{1}$. Thus, $R H_{1}=A_{\infty}$ as sets. Moreover, the constants are related by

$$
\frac{1}{e} R H_{1}(\omega) \leq A_{\infty}(\omega) \leq C \frac{e^{e^{R H_{1}(\omega)}}}{e^{R H_{1}(\omega)}},
$$

where $C$ is independent of $R H_{1}(\omega)$. The constant $1 / e$ is sharp, and the right-hand inequality is sharp in $R H_{1}(\omega)$. The same proofs go through for the dyadic case. We note that $A_{\infty}$ is the union of the $A_{p}$ classes, which are nested and increasing 
as $p \rightarrow \infty$, and also that $R H_{1}$ is the union of the $R H_{p}$ classes, which are nested and decreasing as $p \rightarrow \infty$. Thus, $A_{\infty}=\bigcup_{1 \leq p<\infty} A_{p}=\bigcup_{1<p \leq \infty} R H_{p}=R H_{1}$.

The main result of this subsection is as follows.

Theorem 7.1. Suppose $\delta \in \mathbb{R}$ is far from dyadic rationals, in the sense of condition (1.2). Then the following assertions hold:

(a) $\omega$ is a doubling weight if and only if $\omega$ is dyadic doubling with respect to both $\mathcal{D}$ and $\mathcal{D}^{\delta}$.

(b) $A_{p}=A_{p}^{d} \cap A_{p}^{\delta}$, for each $p$ with $1 \leq p \leq \infty$.

(c) $R H_{p}=R H_{p}^{d} \cap R H_{p}^{\delta}$, for each $p$ with $1 \leq p \leq \infty$.

The constant $A_{p}(\omega)$ depends only on $A_{p}^{d}(\omega)$ and $A_{p}^{\delta}(\omega)$, and vice versa, and similarly for the other classes.

Proof. It is immediate that if $\omega$ is doubling then it is dyadic doubling with respect to both $\mathcal{D}$ and $\mathcal{D}^{\delta}$. Similarly, if $\omega$ lies in $A_{p}$ then $\omega$ lies in both $A_{p}^{d}$ and $A_{p}^{\delta}$ with $\max \left\{A_{p}^{d}(\omega), A_{p}^{\delta}(\omega)\right\} \leq A_{p}(\omega)$, and analogously for $R H_{p}$. Thus, we need only prove the reverse inclusions $(\supset)$.

(a) Suppose $\omega$ is dyadic doubling with respect to both $\mathcal{D}$ and $\mathcal{D}^{\delta}$. Take an interval $Q$ in $\mathbb{R}$. The double $\widetilde{Q}$ of $Q$ is the interval $\widetilde{Q}$ that has the same midpoint as $Q$ and twice the length: $|\widetilde{Q}|=2|Q|$. Let $I$ be an interval of the type guaranteed by Proposition 2.1 applied to $\widetilde{Q}$. Take $N$ such that $2^{N-1} \leq C(\delta)<2^{N}$. Then $|I| \leq 2^{N}|\widetilde{Q}|$

The dyadic subintervals $J$ of $I$ at scale $|J|=2^{-N-2}|I|$ satisfy $|J| \leq|Q| / 2$, which implies that $Q$ contains at least one such $J$. Now we have

$$
\int_{\widetilde{Q}} \omega \leq \int_{I} \omega \leq C_{\mathrm{dy}}^{N+2} \int_{J} \omega \leq C_{\mathrm{dy}}^{N+2} \int_{Q} \omega \leq C_{\mathrm{dy}}^{\log _{2}\left(2^{3} C(\delta)\right)} \int_{Q} \omega .
$$

Thus $\omega$ is doubling, with doubling constant at most $C_{\mathrm{dy}}^{\log _{2}\left(2^{3} C(\delta)\right)}$.

(b) First suppose $1<p<\infty$. The $A_{p}$ weights $\omega$ are characterized by the boundedness of the maximal operator $M$ on $L^{p}(\omega)$, and similarly for dyadic $A_{p}^{d}$ weights and the dyadic maximal operator $M_{d}$. Suppose $\omega \in A_{p}^{d} \cap A_{p}^{\delta}$. Then by Proposition 6.1, for each $f \in L^{p}$ we have

$$
\|M f\|_{L^{p}(\omega)} \leq C(\delta)\left\|M_{d} f+M_{\delta} f\right\|_{L^{p}(\omega)} \leq C C(\delta)\|f\|_{L^{p}(\omega)} .
$$

Therefore $\omega \in A_{p}$.

Now suppose $p=1$. The $A_{1}$ weights $\omega$ are characterized by the existence of a constant $C$ such that $M \omega(x) \leq C \omega(x)$ for almost all $x$, and similarly for dyadic $A_{p}^{d}$ weights and $M_{d} \omega(x) \leq C^{\prime} \omega(x)$. Take $\omega \in A_{1}^{d} \cap A_{1}^{\delta}$. Then by Proposition 6.1 , we have

$$
M \omega(x) \leq C(\delta)\left(M_{d} \omega(x)+M_{\delta} \omega(x)\right) \leq 2 C^{\prime} C(\delta) \omega(x)
$$

for almost all $x$. Thus $\omega$ is in $A_{1}$. 
For $p=\infty$, if $\omega \in A_{\infty}^{d} \cap A_{\infty}^{\delta}$, then $\omega \in A_{p_{1}}^{d} \cap A_{p_{2}}^{\delta}$ for some $p_{1}, p_{2} \in[1, \infty)$. Let $p=\max \left\{p_{1}, p_{2}\right\}$. Then $\omega \in A_{p}^{d} \cap A_{p}^{\delta}$, so by the cases $1 \leq p<\infty$ above, we have $\omega \in A_{p} \subset A_{\infty}$.

(c) We turn to the reverse-Hölder class $R H_{p}$. First, a short calculation shows that for $1 \leq p<\infty$ the following equivalence holds:

$$
\omega \in R H_{p^{\prime}}(d \mu) \Longleftrightarrow \frac{1}{\omega} \in A_{p}(\omega d \mu),
$$

where $A_{p}(\omega d \mu)$ denotes the $A_{p}$ class defined with respect to the measure $\omega d \mu$. Here $\mu$ is a Borel measure, $\omega$ is a nonnegative function such that $\omega$ and $1 / \omega$ are locally integrable, and as usual $p^{\prime}$ is the dual exponent to $p$. See also pp. 693-694 of [13]. The dyadic version of equivalence (7.1) also holds; the only difference is that the suprema are taken over only the dyadic intervals.

Next we establish a more general version of our $A_{p}$ intersection result Theorem 7.1 (b). In this new weighted $A_{p}$ version, the $A_{p}$ class is itself weighted by an $A_{\infty}$ weight.

Lemma 7.2. Let $v$ be an $A_{\infty}$ weight, let $\mu$ be Lebesgue measure, suppose $\delta \in \mathbb{R}$ is far from dyadic rationals in the sense of condition (1.2), and take $p$ with $1 \leq p \leq \infty$. Then $A_{p}(v d \mu)=A_{p}^{d}(v d \mu) \cap A_{p}^{\delta}(v d \mu)$.

Proof of Lemma 7.2. We follow the proof of the unweighted version above; we need only establish a weighted version of Proposition 2.1. In the weighted Proposition 2.1, the only difference is in part (ii) of the conclusion. Take $\delta, Q$, and $I$ as in Proposition 2.1. Since $v \in A_{\infty}$, we have $v \in A_{p}$ for some $p \in[1, \infty)$. If $p=1$, then

$$
\frac{v(I)}{v(Q)} \leq C_{1} \frac{|I|}{|Q|},
$$

where $C_{1}$ can be taken to be a constant such that $M v(x) \leq C_{1} v(x)$ for a.e. $x$. See the discussion on pp. 677-678 of [13]. On the other hand, if $1<p<\infty$, then

$$
\frac{v(I)}{v(Q)} \leq A_{p}(v)\left(\frac{|I|}{|Q|}\right)^{p},
$$

by Exercise 9.2 .5 (b) on p. 693 of [13]. In either case, since by Proposition 2.1 $|I| /|Q|$ is bounded by $C(\delta)$, we find that $v(I) / v(Q)$ is bounded by a constant that depends only on $\delta$ and on $v$. Thus the desired weighted version of condition (ii) holds.

Now, suppose $\delta$ is far from dyadic rationals, and suppose $1<p^{\prime} \leq \infty$. Let $\mu$ be Lebesgue measure. Take $\omega \in R H_{p^{\prime}}^{d}(d \mu) \cap R H_{p^{\prime}}^{\delta}(d \mu)$. Then $\omega$ is in $A_{\infty}^{d}(d \mu) \cap$ $A_{\infty}^{\delta}(d \mu)$, which implies that $\omega \in A_{\infty}(d \mu)$ by Theorem $7.1(\mathrm{~b})$. Therefore our weighted version of Theorem $7.1(\mathrm{~b})$, for $A_{p}(v d \mu)$, holds with the choice $v=\omega$.

Next, by the dyadic version of equivalence (7.1), we have $1 / \omega \in A_{p}^{d}(\omega d \mu) \cap$ $A_{p}^{\delta}(\omega d \mu)$. Then $1 / \omega \in A_{p}(\omega d \mu)$, by Theorem 7.1 (b) adapted to the weighted class $A_{p}(\omega d \mu)$. Therefore $\omega \in R H_{p^{\prime}}(d \mu)$, by equivalence (7.1). 
Finally, for $p^{\prime}=1$, suppose $\omega$ belongs to both $R H_{1}^{d}$ and $R H_{1}^{\delta}$. By the comment about $R H_{1}$ before Theorem 7.1 above, $\omega$ belongs to both $A_{\infty}^{d}$ and $A_{\infty}^{\delta}$, with constants depending only on $R H_{1}^{d}(\omega)$ and $R H_{1}^{\delta}(\omega)$. So by part (b) above, $\omega$ belongs to $A_{\infty}$, and by the same comment, $\omega$ belongs to $R H_{1}$, with constant $R H_{1}(\omega)$ depending only on $R H_{1}^{d}(\omega)$ and $R H_{1}^{\delta}(\omega)$.

We note that Theorem 7.1 (b) and (c) can also be proved without reference to the characterization of $A_{p}$ via the maximal function, but rather from the definitions of $A_{p}$ and $R H_{p}$ via integral conditions, together with Lemma 2.2. These proofs lead to the following bounds on the constants:

$$
\begin{aligned}
A_{p}(\omega) & \leq C(\delta)^{p} \max \left\{A_{p}^{d}(\omega), A_{p}^{\delta}(\omega)\right\}, \quad \text { for } 1 \leq p<\infty, \quad \text { and } \\
R H_{p}(\omega) & \leq C(\delta)^{1 / p}\left(C_{\mathrm{dy}}\right)^{\log _{2}(4 C(\delta))} \max \left\{R H_{p}^{d}(\omega), R H_{p}^{\delta}(\omega)\right\}, \quad \text { for } 1<p \leq \infty .
\end{aligned}
$$

Also, the constants $A_{\infty}(\omega)$ and $R H_{1}(\omega)$ depend only on $\max \left\{A_{\infty}^{d}(\omega), A_{\infty}^{\delta}(\omega)\right\}$ and $\max \left\{R H_{1}^{d}(\omega), R H_{1}^{\delta}(\omega)\right\}$, respectively.

\subsection{Multiparameter doubling, $A_{p}$, and $R H_{p}$ weights}

We extend the above results to multiparameter $A_{p}, R H_{p}$ and doubling weights. This extension is fairly straightforward, since (unlike the proofs above for BMO, $\mathrm{VMO}$ and $H^{1}$ ) it proceeds by iteration of the one-parameter case. As usual, we work in the biparameter case.

The theory of product weights was developed by K.-C. Lin in his thesis [18], while the dyadic theory was developed in Buckley's paper [4]. The product $A_{p}$ and $R H_{p}$ weights (resp. doubling weights) and their dyadic analogues are defined via integral conditions (resp. doubling conditions) parallel to those for the oneparameter case, using rectangles in $\mathbb{R} \otimes \mathbb{R}$ instead of intervals in $\mathbb{R}$. It follows that a product weight belongs to $A_{p}(\mathbb{R} \otimes \mathbb{R})$ if and only if it belongs to $A_{p}(\mathbb{R})$ in each variable separately.

To be precise, $\omega \in A_{p}(\mathbb{R} \otimes \mathbb{R})$ if and only if $\omega(\cdot, y) \in A_{p}(\mathbb{R})$ uniformly for a.e. $y \in \mathbb{R}$ and $\omega(x, \cdot) \in A_{p}(\mathbb{R})$ uniformly for a.e. $x \in \mathbb{R}$. In one direction this fact is a consequence of the Lebesgue differentiation theorem, letting one side of the rectangle shrink to a point. The converse uses the equivalence between $\omega \in A_{p}(\mathbb{R} \otimes \mathbb{R})$ and the maximal inequality for the strong maximal function; see p. 83 of $[26]$. Further, the $A_{p}(\mathbb{R} \otimes \mathbb{R})$ constant depends only on the two $A_{p}(\mathbb{R})$ constants, and vice versa.

The analogous characterizations in terms of the separate variables hold for product $R H_{p}$ and product doubling weights, and for dyadic product $A_{p}, R H_{p}$, and doubling weights.

We denote by $A_{p}^{d, d}=A_{p}^{d, d}(\mathbb{R} \otimes \mathbb{R})$ the class of dyadic product weights, meaning the weights $\omega(x, y)$ such that

(i) for a.e. fixed $y, \omega(\cdot, y)$ lies in $A_{p}^{d}(\mathbb{R})$, and

(ii) for a.e. fixed $x, \omega(x, \cdot)$ lies in $A_{p}^{d}(\mathbb{R})$,

with uniform $A_{p}^{d}(\mathbb{R})$ constants. Define $A_{p}^{d, \delta}$ similarly, using $\mathcal{D}^{\delta}$ instead of $\mathcal{D}$ in the second variable, and similarly for $A_{p}^{\delta, d}, A_{p}^{\delta, \delta}$, and the four variants of $R H_{p}^{d, d}$. 
Theorem 7.3. Suppose $\delta \in \mathbb{R}$ is far from dyadic rationals, in the sense of condition (1.2). Then the following assertions hold:

(a) A weight $\omega(x, y)$ is a product doubling weight if and only if $\omega$ is dyadic doubling with respect to each of $\mathcal{D} \times \mathcal{D}, \mathcal{D} \times \mathcal{D}^{\delta}, \mathcal{D}^{\delta} \times \mathcal{D}$, and $\mathcal{D}^{\delta} \times \mathcal{D}^{\delta}$.

(b) For $1 \leq p \leq \infty$, biparameter $A_{p}$ is the intersection of four translates of biparameter dyadic $A_{p}$ :

$$
A_{p}(\mathbb{R} \otimes \mathbb{R})=A_{p}^{d, d}(\mathbb{R} \otimes \mathbb{R}) \cap A_{p}^{d, \delta}(\mathbb{R} \otimes \mathbb{R}) \cap A_{p}^{\delta, d}(\mathbb{R} \otimes \mathbb{R}) \cap A_{p}^{\delta, \delta}(\mathbb{R} \otimes \mathbb{R}) .
$$

(c) For $1 \leq p \leq \infty$, biparameter $R H_{p}$ is the intersection of four translates of biparameter dyadic $\mathrm{RH}_{p}$ :

$$
R H_{p}(\mathbb{R} \otimes \mathbb{R})=R H_{p}^{d, d}(\mathbb{R} \otimes \mathbb{R}) \cap R H_{p}^{d, \delta}(\mathbb{R} \otimes \mathbb{R}) \cap R H_{p}^{\delta, d}(\mathbb{R} \otimes \mathbb{R}) \cap R H_{p}^{\delta, \delta}(\mathbb{R} \otimes \mathbb{R}) .
$$

The constant $A_{p}(\omega)$ depends only on $A_{p}^{d, d}(\omega), A_{p}^{d, \delta}(\omega), A_{p}^{\delta, d}(\omega)$, and $A_{p}^{\delta, \delta}(\omega)$, and vice versa, and similarly for the other classes.

Proof. The proof is by iteration of the one-parameter argument. We sketch the case of $A_{p}$ for $1<p<\infty$. The other cases are similar. Take $\omega \in A_{p}(\mathbb{R} \otimes \mathbb{R})$. By the characterization of $A_{p}(\mathbb{R} \otimes \mathbb{R})$ above, together with the one-parameter result (Theorem 7.1), we have $\omega(\cdot, y) \in A_{p}(\mathbb{R})=A_{p}^{d}(\mathbb{R}) \cap A_{p}^{\delta}(\mathbb{R})$ uniformly for almost every $y$, and similarly in the other variable. Thus $\omega \in A_{p}^{d, d}(\mathbb{R} \otimes \mathbb{R})$, and similarly $\omega \in A_{p}^{d, \delta}(\mathbb{R} \otimes \mathbb{R}), \omega \in A_{p}^{\delta, d}(\mathbb{R} \otimes \mathbb{R})$, and $\omega \in A_{p}^{\delta, \delta}(\mathbb{R} \otimes \mathbb{R})$. Conversely, given $\omega$ is in the intersection of the four dyadic spaces, we see that $\omega(\cdot, y)$ belongs to $A_{p}^{d}(\mathbb{R}) \cap A_{p}^{\delta}(\mathbb{R})=A_{p}(\mathbb{R})$ uniformly for a.e. $y$, and similarly in the other variable. Therefore $\omega \in A_{p}(\mathbb{R} \otimes \mathbb{R})$. Moreover, the claimed dependence of the constants follows immediately from the one-parameter result.

\section{Weighted dyadic structure theorems}

In this section we present our results on weighted Hardy spaces and on weighted maximal functions. As usual, we present the biparameter case. We also note that during the proof of Theorem 7.1 (c) above, we have established a weighted version (Lemma 7.2) of the dyadic structure theorem for $A_{p}$ (Theorem $7.1(\mathrm{~b})$ ).

\subsection{Weighted Hardy spaces}

Suppose $\omega \in A_{\infty}$ is a doubling weight. Denote the weighted Hardy space by $H_{\omega}^{1}(\mathbb{R})$. Similarly, denote by $H_{d, \omega}^{1}(\mathbb{R})$ (resp. $H_{\delta, \omega}^{1}(\mathbb{R})$ ) the weighted dyadic (resp. weighted $\delta$-dyadic) Hardy space with respect to $\mathcal{D}\left(\operatorname{resp} . \mathcal{D}^{\delta}\right)$.

It is shown in [30] that if $\omega$ is an $A_{\infty}$ weight, then the definitions of $H_{\omega}^{1}(\mathbb{R})$ via the weighted square function and via the atomic decomposition are equivalent, with equivalent norms, and the dual of $H_{\omega}^{1}(\mathbb{R})$ is the weighted space $\mathrm{BMO}_{\omega}(\mathbb{R})$. The same is true in the dyadic case. 
In parallel with the one-parameter case, given a product $A_{\infty}$ weight $\omega$ we define the weighted product Hardy spaces $H_{\omega}^{1}(\mathbb{R} \otimes \mathbb{R})$ and the dyadic versions $H_{d, d, \omega}^{1}(\mathbb{R} \otimes \mathbb{R}), H_{d, \delta, \omega}^{1}(\mathbb{R} \otimes \mathbb{R}), H_{\delta, d, \omega}^{1}(\mathbb{R} \otimes \mathbb{R})$, and $H_{\delta, \delta, \omega}^{1}(\mathbb{R} \otimes \mathbb{R})$. As in the oneparameter case, it can be shown that the definition in terms of the weighted square function is equivalent to the definition in terms of weighted product atoms, and that the dual of product weighted $H^{1}$ is product weighted BMO, in both the continuous and dyadic cases. We omit the details.

Theorem 8.1. Suppose $\delta \in \mathbb{R}$ is far from dyadic rationals, in the sense of condition (1.2). Suppose $\omega$ is an $A_{\infty}$ weight. Then the following relations hold:

(i) $H_{\omega}^{1}(\mathbb{R})=H_{d, \omega}^{1}(\mathbb{R})+H_{\delta, \omega}^{1}(\mathbb{R})$, with equivalent norms.

(ii) $H_{\omega}^{1}(\mathbb{R} \otimes \mathbb{R})=H_{d, d, \omega}^{1}(\mathbb{R} \otimes \mathbb{R})+H_{d, \delta, \omega}^{1}(\mathbb{R} \otimes \mathbb{R})+H_{\delta, d, \omega}^{1}(\mathbb{R} \otimes \mathbb{R})+H_{\delta, \delta, \omega}^{1}(\mathbb{R} \otimes \mathbb{R})$, with equivalent norms.

Proof. For the one-parameter case, recall that a function $a$ is an atom of the weighted Hardy space $H_{\omega}^{1}(\mathbb{R})$ if it satisfies

(a) $\operatorname{supp} a \subset Q$ for some interval $Q \subset \mathbb{R}$;

(b) $\|a\|_{L^{2}(\omega)} \leq \omega(Q)^{-1 / 2}$; and

(c) $\int a(x) d x=0$.

Similarly, the atoms of $H_{d, \omega}^{1}(\mathbb{R})$ (resp. $H_{\delta, \omega}^{1}(\mathbb{R})$ ) satisfy the same conditions (a), (b) and (c) above with the extra condition that $Q \in \mathcal{D}$ (resp. $Q \in \mathcal{D}^{\delta}$ ).

From the definitions of atoms, it is immediate that each $H_{d, \omega}^{1}(\mathbb{R})$-atom or $H_{\delta, \omega}^{1}(\mathbb{R})$-atom is an $H_{\omega}^{1}(\mathbb{R})$-atom. Thus, $H_{d, \omega}^{1}(\mathbb{R}) \subset H_{\omega}^{1}(\mathbb{R})$ and $H_{\delta, \omega}^{1}(\mathbb{R}) \subset H_{\omega}^{1}(\mathbb{R})$ with norms $\|f\|_{H_{\omega}^{1}(\mathbb{R})} \leq\|f\|_{H_{d, \omega}^{1}(\mathbb{R})}$ and $\|f\|_{H_{\omega}^{1}(\mathbb{R})} \leq\|f\|_{H_{\delta, \omega}^{1}(\mathbb{R})}$, and so $H_{\omega}^{1}(\mathbb{R}) \supset$ $H_{d, \omega}^{1}(\mathbb{R})+H_{\delta, \omega}^{1}(\mathbb{R})$.

For the converse, suppose $a$ is an $H_{\omega}^{1}(\mathbb{R})$-atom satisfying (a), (b) and (c) with an interval $Q$. Then, by Proposition 2.1, there exists an interval $I$ such that $Q \subset I$, $|I| \leq C(\delta)|Q|$ and either $I \in \mathcal{D}$ or $I \in \mathcal{D}^{\delta}$. Moreover, Lemma 2.2 implies that

$$
\|a\|_{L^{2}(\omega)} \leq \omega(Q)^{-1 / 2} \leq C(\delta)^{1 / 2}\left(C_{\mathrm{dy}}\right)^{\frac{1}{2} \log _{2}(4 C(\delta))} \omega(I)^{-1 / 2} .
$$

Let $C_{0}:=C(\delta)^{1 / 2}\left(C_{\mathrm{dy}}\right)^{\frac{1}{2} \log _{2}(4 C(\delta))}$. Then the above inequality implies that $C_{0}^{-1} a$ is an $H_{d, \omega}^{1}(\mathbb{R})$-atom if $I \in \mathcal{D}$, and an $H_{\delta, \omega}^{1}(\mathbb{R})$-atom if $I \in \mathcal{D}^{\delta}$. It follows that $H_{\omega}^{1}(\mathbb{R}) \subset H_{d, \omega}^{1}(\mathbb{R})+H_{\delta, \omega}^{1}(\mathbb{R})$ with norms $\|f\|_{H_{d, \omega}^{1}(\mathbb{R})}+\|f\|_{H_{\delta, \omega}^{1}(\mathbb{R})} \leq C_{0}\|f\|_{H_{\omega}^{1}(\mathbb{R})}$.

The product case is similar. We omit the details.

\subsection{Weighted maximal functions}

Let $\omega$ be a doubling weight. For $f \in L_{\text {loc }}^{1}(\mathbb{R}, \omega)$, denote by $M_{\omega}(f)$ the weighted Hardy-Littlewood maximal function, defined by

$$
M_{\omega}(f)(x):=\sup _{Q \ni x} \frac{1}{\omega(Q)} \int_{Q}|f(y)| \omega(y) d y
$$


where the supremum is taken over all intervals $Q \subset \mathbb{R}$ that contain $x$. Similarly, denote by $M_{d, \omega}(f)\left(\operatorname{resp} . M_{\delta, \omega}(f)\right)$ the dyadic (resp. $\delta$-dyadic) weighted maximal function; here $\delta \in \mathbb{R}$ and the supremum is taken over only those intervals $I \in \mathcal{D}$ (resp. $I \in \mathcal{D}^{\delta}$ ) that contain $x$.

In the multiparameter setting, for a product doubling weight $\omega(x, y)$ we consider the weighted strong maximal function defined for $f \in L_{\text {loc }}^{1}(\mathbb{R} \otimes \mathbb{R}, \omega)$ by

$$
M_{s, \omega}(f)(x, y):=\sup _{R \ni(x, y)} \frac{1}{|R|} \int_{R}|f(u, v)| \omega(u, v) d u d v
$$

where the supremum is over all rectangles $R \subset \mathbb{R} \otimes \mathbb{R}$ that contain $(x, y)$, and its dyadic versions $M_{s, \omega}^{d, d}(f), M_{s, \omega}^{d, \delta}(f), M_{s, \omega}^{\delta, d}(f)$ and $M_{s, \omega}^{\delta, \delta}(f)$, formed by restricting the supremum to be over rectangles containing $(x, y)$ that lie in $\mathcal{D} \times \mathcal{D}, \mathcal{D} \times \mathcal{D}^{\delta}$, $\mathcal{D}^{\delta} \times \mathcal{D}$, and $\mathcal{D}^{\delta} \times \mathcal{D}^{\delta}$, respectively.

Theorem 8.2. Suppose $\delta \in \mathbb{R}$ is far from dyadic rationals, in the sense of condition (1.2). Then the following relations hold, with implicit constants independent of $f$ :

(i) For each doubling weight $\omega(x)$ and for each $f \in L_{\mathrm{loc}}^{1}(\mathbb{R}, \omega), M_{\omega}(f)$ is pointwise equivalent to $M_{d, \omega}(f)+M_{\delta, \omega}(f)$.

(ii) For each product doubling weight $\omega(x, y)$ and for each $f \in L_{\mathrm{loc}}^{1}(\mathbb{R} \otimes \mathbb{R}, \omega)$, $M_{s, \omega}(f)$ is pointwise equivalent to $M_{s, \omega}^{d, d}(f)+M_{s, \omega}^{d, \delta}(f)+M_{s, \omega}^{\delta, d}(f)+M_{s, \omega}^{\delta, \delta}(f)$.

Proof. It is immediate that $M_{d, \omega}(f) \leq M_{\omega}(f)$ and $M_{\delta, \omega}(f) \leq M_{\omega}(f)$ for every $f \in L_{\text {loc }}^{1}(\mathbb{R})$. Conversely, for each interval $Q \subset \mathbb{R}$, by Proposition 2.1, there is an interval $I$ such that $Q \subset I,|I| \leq C(\delta)|Q|$ and $I \in \mathcal{D}$ or $I \in \mathcal{D}^{\delta}$. Moreover, from Lemma 2.2 we have

$$
\omega(I) \leq\left(C_{\mathrm{dy}}\right)^{\log _{2}(4 C(\delta))} \omega(Q) \frac{|I|}{|Q|} \leq C(\delta)\left(C_{\mathrm{dy}}\right)^{\log _{2}(4 C(\delta))} \omega(Q) .
$$

Consequently, we obtain that

$$
\frac{1}{\omega(Q)} \int_{Q}|f(y)| \omega(y) d y \leq C(\delta)\left(C_{\mathrm{dy}}\right)^{\log _{2}(4 C(\delta))} \frac{1}{\omega(I)} \int_{I}|f(y)| \omega(y) d y,
$$

which implies that $M_{\omega}(f) \leq C(\delta)\left(C_{\mathrm{dy}}\right)^{\log _{2}(4 C(\delta))}\left(M_{\delta, \omega}(f)+M_{d, \omega}(f)\right)$.

The multiparameter proof is similar. We omit the details.

\section{References}

[1] Bernard, A.: Espaces $H^{1}$ de martingales à deux indices. Dualité avec les martingales de type "BMO" (French). Bull. Sci. Math. (2) 103 (1979), no. 3, 297-303.

[2] Beznosova, O. And Reznikov, A.: Sharp estimates involving $A_{\infty}$ and $L \log L$ constants, and their applications to PDE. Algebra i Analiz 26 (2014), no. 1, 40-67; translation in St. Petersburg Math. J. 26 (2015), no. 1, 27-47. 
[3] Bourdaud, G.: Remarques sur certains sous-espaces de $\operatorname{BMO}\left(\mathbb{R}^{n}\right)$ et de $\operatorname{bmo}\left(\mathbb{R}^{n}\right)$. Ann. Inst. Fourier (Grenoble) 52 (2002), 1187-1218.

[4] Buckley, S. M.: Summation conditions on weights. Michigan Math. J. 40 (1993), 153-170.

[5] Chang, S.-Y.A. And Fefferman, R.: A continuous version of duality of $H^{1}$ with BMO on the bidisc. Ann. of Math. (2) 112 (1980), no. 1, 179-201.

[6] Chen, P., Li, J. And WArd, L. A.: BMO from dyadic BMO via expectations on product spaces of homogeneous type., J. Funct. Anal. 265 (2013), 2420-2451.

[7] Coifman, R. R. and Weiss, G.: Extensions of Hardy spaces and their use in analysis. Bull. Amer. Math. Soc.: 83 (1977), 569-645.

[8] Cruz-Uribe, D. And Neugebauer, C. J.: The structure of the reverse Hölder classes. Trans. Amer. Math. Soc. 347 (1995), 2941-2960.

[9] Deng, D. G., Duong, X. T., Song, L., Tan, C. Q. and Yan, L. X.: Functions of vanishing mean oscillation associated with operators and applications. Michigan Math. J. 56 (2008), 529-550.

[10] García-Cuerva, J. And Rubio de Francia, J. L.: Weighted norm inequalities and related topics. North-Holland Mathematics Studies 16, North-Holland, Amsterdam, 1985.

[11] Garnett, J. B.: Bounded analytic functions. Pure and Applied Mathematics 96, Academic Press, New York-London, 1981.

[12] Garnett, J. B. And Jones, P. W.: BMO from dyadic BMO. Pacific J. Math. 99 (1982), no. 2, 351-371.

[13] Grafakos, L.: Classical and modern Fourier analysis. Pearson Education, Upper Saddle River, NJ, 2004.

[14] Hytönen, T. and PÉrez, C.: Sharp weighted bounds involving $A_{\infty}$. Anal. PDE 6 (2013), no. 4, 777-818.

[15] Han, Y., Li, J. And Lu, G.: Duality of multiparameter Hardy space $H^{p}$ on product spaces of homogeneous type. Ann. Sc. Norm. Super. Pisa Cl. Sci. (5) 9 (2010), 645-685.

[16] John, F. And Nirenberg, L.: On functions of bounded mean oscillation. Comm. Pure Appl. Math. 14 (1961), 415-426.

[17] Lacey, M. T., Terwilleger, E. And Wick, B.: Remarks on product VMO. Proc. Amer. Math. Soc. 134 (2006), no. 2, 465-474.

[18] Lin, K.-C.: Harmonic analysis on the bidisc. Ph. D. thesis, UCLA, 1984.

[19] MeI, T.: BMO is the intersection of two translates of dyadic BMO. C. R. Math. Acad. Sci. Paris 336 (2003), no. 12, 1003-1006.

[20] Muckenhoupt, B.: Weighted norm inequalities for the Hardy maximal function. Trans. Amer. Math. Soc. 165 (1972), 207-226.

[21] Okikiolu, K.: Characterization of subsets of rectifiable curves in $\mathbb{R}^{n}$. J. London Math. Soc. (2) 46 (1992), 336-348.

[22] Pipher, J.: Double index square functions and bounded mean oscillation on the bidisc. Ph. D. thesis, UCLA, 1985.

[23] Pipher, J. And WARD, L. A.: BMO from dyadic BMO on the bidisc. J. London Math. Soc. 77 (2008), 524-544. 
[24] Pipher, J., Ward, L. A. And XiaO, X.: Geometric-arithmetic averaging of dyadic weights. Rev. Mat. Iberoamericana 27 (2011), no. 3, 953-976.

[25] Sarason, D.: Functions of vanishing mean oscillation. Trans. Amer. Math. Soc. 207 (1975), 391-405.

[26] Stein, E. M.: Harmonic analysis: real-variable methods, orthogonality, and oscillatory integrals. Princeton Mathematical Series 43, Monographs in Harmonic Analysis III, Princeton University Press, Princeton, NJ, 1993.

[27] Treil, S.: $H^{1}$ and dyadic $H^{1}$. In Linear and complex analysis: Dedicated to V.P. Havin on the occasion of his 75th birthday (ed. S. Kislyakov, A. Alexandrov, A. Baranov), 179-194. Advances in the Mathematical Sciences 226, American Mathematical Society, 2009 .

[28] Uchiyama, A.: On the compactness of operators of the Hankel type. Tôhoku Math. J. (2) 30 (1978), 163-171.

[29] Ward, L. A.: Translation averages of dyadic weights are not always good weights. Rev. Mat. Iberoamericana 18 (2002), no. 2, 379-407.

[30] Wu, S.: A wavelet characterization for weighted Hardy spaces. Rev. Mat. Iberoamericana 8 (1992), no. 3, 329-349.

Received June 11, 2013.

Ji Li: Department of Mathematics, Macquarie University, NSW, 2109, Australia.

E-mail: ji.li@mq.edu.au

Jill PIPHer: Department of Mathematics, Brown University, Providence, RI 02912, USA.

E-mail: jpipher@math.brown.edu

Lesley A. WARD: School of Information Technology and Mathematical Sciences, University of South Australia, Mawson Lakes SA 5095, Australia.

E-mail: Lesley.Ward@unisa.edu.au

The authors were supported by the Australian Research Council, Grant No. ARC-DP120100399. The first author was also supported by a University of South Australia postdoctoral fellowship, by the NNSF of China, Grant No. 11001275, by a China Postdoctoral Science Foundation funded project, Grant No. 201104383, and by the Fundamental Research Funds for the Central Universities, Grant No. 11lgpy56. The third author was also supported by the NSF, Grant No. DMS0901139. 